\title{
ARTICLE
}

\section{Regulation of monocyte cell fate by blood vessels mediated by Notch signalling}

Jaba Gamrekelashvili , Roberto Giagnorio ${ }^{1,2}$, Jasmin Jussofie ${ }^{3, \dagger}$, Oliver Soehnlein 4,5,6, Johan Duchene ${ }^{4}$, Carlos G. Briseño ${ }^{7}$, Saravana K. Ramasamy ${ }^{8}$, Kashyap Krishnasamy ${ }^{1}$, Anne Limbourg ${ }^{9}$, Christine Häger ${ }^{3, \dagger}$, Tamar Kapanadze ${ }^{1,2}$, Chieko Ishifune ${ }^{10}$, Rabea Hinkel ${ }^{4,11}$, Freddy Radtke ${ }^{12}$, Lothar J. Strobl $\left.\right|^{13}$, Ursula Zimber-Strobl ${ }^{13}$, L. Christian Napp ${ }^{3}$, Johann Bauersachs ${ }^{3}$, Hermann Haller ${ }^{1}$, Koji Yasutomo ${ }^{10}$, Christian Kupatt ${ }^{4,11}$, Kenneth M. Murphy ${ }^{7,14}$, Ralf H. Adams ${ }^{8}$, Christian Weber ${ }^{4,15}$ \& Florian P. Limbourg ${ }^{1,2}$

A population of monocytes, known as Ly6Clo monocytes, patrol blood vessels by crawling along the vascular endothelium. Here we show that endothelial cells control their origin through Notch signalling. Using combinations of conditional genetic deletion strategies and cell-fate tracking experiments we show that Notch2 regulates conversion of Ly6Chi monocytes into Ly $6 \mathrm{C}^{\mathrm{lo}}$ monocytes in vivo and in vitro, thereby regulating monocyte cell fate under steady-state conditions. This process is controlled by Notch ligand delta-like 1 (DII1) expressed by a population of endothelial cells that constitute distinct vascular niches in the bone marrow and spleen in vivo, while culture on recombinant DLL1 induces monocyte conversion in vitro. Thus, blood vessels regulate monocyte conversion, a form of committed myeloid cell fate regulation.

\footnotetext{
${ }^{1}$ Department of Nephrology and Hypertension, Hannover Medical School, D 30625 Hannover, Germany. ${ }^{2}$ Integrated Research and Treatment Center Transplantation, Hannover Medical School, D 30625 Hannover, Germany. ${ }^{3}$ Department of Cardiology, Hannover Medical School, D 30625 Hannover, Germany. ${ }^{4}$ Institute for Cardiovascular Prevention, Ludwig-Maximilians-University, D 80336 Munich, Germany. ${ }^{5}$ Academic Medical Center, Department of Pathology, Amsterdam University, 1105 AZ Amsterdam, The Netherlands. ${ }^{6}$ German Centre for Cardiovascular Research, Partner Site Munich Heart Alliance, Munich, Germany. ${ }^{7}$ Department of Pathology and Immunology, Washington University in St Louis, School of Medicine, St Louis, Missouri 63110, USA. ${ }^{8}$ Max Planck Institute for Molecular Biomedicine, D 48149 Muenster, Germany. ${ }^{9}$ Department of Plastic, Hand and Reconstructive Surgery, Hannover Medical School, D 30625 Hannover, Germany. ${ }^{10}$ Department of Immunology and Parasitology, Graduate School of Medicine, Tokushima University, Tokushima 770-8503, Japan. ${ }^{11}$ Medizinische Klinik I, Klinikum Rechts der Isar, Technical University of Munich, D 81675 Munich, Germany. ${ }^{12}$ Ecole Polytechnique Fédérale de Lausanne, School of Life Sciences, ISREC, CH 1015 Lausanne, Switzerland. ${ }^{13}$ Research Unit Gene Vectors, Helmholtz Zentrum München, German Research Center for Environment and Health (GmbH), D 81377 Munich, Germany. ${ }^{14}$ Howard Hughes Medical Institute, Washington University in St Louis, School of Medicine, St Louis, Missouri, USA. ${ }^{15}$ Cardiovascular Research Institute Maastricht (CARIM), 6229 ER Maastricht, The Netherlands. $\dagger$ †resent addresses: Department of Cardiology, Universitätsklinikum Düsseldorf, D 40225 Düsseldorf, Germany (J.S.); Institute for Laboratory Animal Science and Central Animal Facility, Hannover Medical School, Hannover D 30625, Germany (C.H.). Correspondence and requests for materials should be addressed to F.P.L. (email: limbourg.florian@mh-hannover.de).
} 
$\mathrm{M}$ onocytes are myeloid leukocytes that circulate through blood vessels and patrol the vascular endothelium or differentiate into mononuclear phagocytes. Mouse monocytes consist of two distinct subsets that differ in behaviour and function, which can be discriminated by expression of the Ly6C antigen and $\mathrm{CX}_{3} \mathrm{CR} 1$ chemokine receptor in combination with additional surface markers ${ }^{1,2}$. So called classical or Ly6 $\mathrm{C}^{\text {hi }}$ monocytes are characterized by $\mathrm{Ly} 6 \mathrm{C}^{\mathrm{hi}} \mathrm{CX}_{3} \mathrm{CR} 1^{\mathrm{lo}}$ and constitute the more prevalent subset. They develop from the recently identified common monocyte progenitor $(\mathrm{cMoP})^{3}$, which in turn is derived from the macrophage and dendritic cell progenitor $(\mathrm{MDP})^{4}$. Under steady-state conditions, Ly6 $\mathrm{C}^{\text {hi }}$ monocytes circulate in the blood for short periods of time and are the definitive precursors of certain tissue-resident mononuclear phagocytes, for example in the gut, skin and spleen $^{2}$. When recruited to sites of inflammation through interaction with subsets of activated vascular endothelial cells (ECs), Ly6C ${ }^{\text {hi }}$ monocytes give rise to macrophages and dendritic cells, produce inflammatory mediators and orchestrate the inflammatory response ${ }^{5-7}$. Based on functional and gene expression studies, these monocytes correspond to a subset of 'classical' human monocytes.

There is a second subtype of monocytes that interacts closely and constitutively with blood vessels. They display a Ly $6 \mathrm{C}^{\mathrm{lo}} \mathrm{CX}_{3} \mathrm{CR} 1^{\text {hi }}$ signature and hence are called $\mathrm{Ly} 6 \mathrm{C}^{\text {lo }}$ monocytes. In the steady-state, Ly6C $\mathrm{C}^{\mathrm{lo}}$ monocytes are long-lived and remain mostly within blood vessels, where they crawl along the luminal side of EC to monitor blood vessels and scavenge microparticles ${ }^{8}$, a feature shared with the human $\mathrm{CD}^{\circ} 6^{+}$ monocyte subset ${ }^{9}$. After endothelial injury in the kidney, Ly6C $\mathrm{C}^{\mathrm{lo}}$ monocytes orchestrate EC necrosis and clearance ${ }^{10}$. Moreover, Ly6 $\mathrm{C}^{\mathrm{lo}}$ monocytes also mediate IgG-dependent effector functions and are involved in immune complexmediated disease $\mathrm{s}^{11,12}$. Ly6 $\mathrm{C}^{\text {lo }}$ monocytes were also suggested to contribute to ischemic tissue repair ${ }^{5}$.

The cellular and molecular context of Ly6C ${ }^{\text {lo }}$ monocyte development is far from clear. Mice deficient for the transcription factor Nur77 (Nr4a1), an orphan nuclear receptor, show reduced frequency and survival of Ly6C $\mathrm{C}^{\text {lo }}$ monocytes ${ }^{13}$. Recent findings further suggest that in the steady state, Ly6 $\mathrm{C}^{\mathrm{lo}}$ monocytes develop from Ly6 $\mathrm{C}^{\text {hi }}$ monocytes ${ }^{2}$. Grafted MDP and cMoP sequentially give rise to Ly6 $\mathrm{C}^{\text {hi }}$ monocytes followed by Ly6 $\mathrm{C}^{\mathrm{lo}}$ monocytes ${ }^{3,6}$. After adoptive transfer of Ly6C ${ }^{\text {hi }}$ monocytes, Ly6C $C^{\text {lo }}$ monocytes are detected in the blood and bone marrow (BM) of recipients, suggesting that Ly6Chi monocytes convert to Ly6Clo monocytes in the circulation ${ }^{7,14}$. However, the tissues and molecular events regulating monocyte conversion are unknown.

Notch signalling is a cell to cell contact-dependent signalling pathway regulating cell fate decisions and inflammatory responses in the immune system ${ }^{15}$. Activation of Notch receptors (Notch1-4 in mammals) is controlled by membranebound Notch ligands of the jagged (Jag) and Delta-like (Dll) gene families, which show different Notch receptor binding affinities and tissue expression patterns, thereby controlling specific Notch signalling outcomes ${ }^{16,17}$. The cellular interactions required for Notch signalling often occur in local tissue microenvironments, or niche, in the BM and spleen ${ }^{18}$. Vascular EC are a specialized component of the niche that maintain and regulate stem cells and their immune cell progeny by providing instructive paracrine cues, known as angiocrine factors, in part through expression of Notch ligands ${ }^{19}$. In the BM niche ECs trigger self-renewal and repopulation of progenitor cells through Notch ligand Jag1 activating Notch1/Notch2 receptors in stem cells ${ }^{20,21}$. Similarly, expansion and aggressiveness of B-cell lymphomas is induced by an angiocrine mechanism involving endothelial Jag1 activating Notch2 in malignant lymphoma cells ${ }^{22}$. Specialized vascular niches are also found in secondary lymphoid organs, such as the marginal zone (MZ) of the spleen, which constitutes an EC interface between lymphoid follicles and the red pulp. Development of MZ B cells and $\mathrm{Esam}^{+}$dendritic cell in the splenic niche is dependent on Notch ligand delta-like 1 (Dll1)-Notch2 signalling ${ }^{23-25}$. Monocytes, which are resident in BM and spleen, express Notch1 and Notch2, and their cell fate is influenced by DLL1 in vitro ${ }^{26}$.

Because of the intricate relationship of Ly6 $\mathrm{C}^{\mathrm{lo}}$ monocytes with EC we reasoned that blood vessels might be involved in monocyte conversion through a Notch-dependent mechanism. We here show that Notch2 signalling regulates conversion of Ly6C $\mathrm{C}^{\text {hi }}$ monocytes into Ly6C $\mathrm{C}^{\mathrm{lo}}$ monocytes, which is controlled by Notch ligand Dll1 expressed by a population of EC present in haematopoietic niches of the BM and spleen. Thus, blood vessels regulate monocyte conversion, a form of committed myeloid cell fate regulation.

\section{Results}

Monocyte populations and lineage relationships. Our aim was to study the regulation of Ly6 $\mathrm{C}^{\mathrm{lo}}$ monocytes. To discriminate monocyte subsets and monocyte progenitor populations in mice we concurrently characterized MDP, cMoP, Ly6 $\mathrm{Ch}^{\text {hi }}$ and Ly6C $\mathrm{C}^{\mathrm{lo}}$ monocytes in $\mathrm{BM}$, spleen and peripheral blood (PB) with common and discriminating markers of monocyte types based on known expression profiles ${ }^{9-3,27}$. This approach was tested in Cx3crl ${ }^{G F P}+$ reporter mice ${ }^{28}$, in which monocyte subsets express distinct intensities of green fluorescent protein (GFP), but also in wild-type mice (Fig. 1a, Supplementary Fig. 1a,b and Supplementary Table 1). In addition, monocyte subpopulations were also validated in Nr4a1-GFP reporter mice ${ }^{29}$, which demonstrated selective GFP expression in Ly6 $\mathrm{C}^{\mathrm{lo}}$ monocytes (Supplementary Fig. 2a,b). These studies confirmed the proto-typical flow cytometry and gene expression profiles reported for Ly6 $\mathrm{C}^{\mathrm{hi}}$ and Ly6C $\mathrm{C}^{\mathrm{lo}}$ monocytes (Fig. $\left.1 \mathrm{~b}, \mathrm{c}\right)^{3}$.

Ly6 $\mathrm{C}^{\mathrm{lo}}$ monocytes are reported to derive from Ly6 $\mathrm{C}^{\text {hi }}$ monocytes under steady-state conditions, based on characteristic changes of two markers, $\mathrm{CX}_{3} \mathrm{CR} 1$ and $\mathrm{Ly} 6 \mathrm{C}$, observed after adoptive transfer of Ly6 $\mathrm{C}^{\text {hi }}$ monocytes ${ }^{7,14}$. To confirm and extend these findings we performed adoptive transfer studies with Ly6C $\mathrm{C}^{\text {hi }}$ monocytes that were isolated from $\mathrm{CD} 45.2^{+} \mathrm{C} \times 3 \mathrm{cr} 1^{\mathrm{GFP} /+}$ mice and intravenously transferred into $\mathrm{CD} 45.1^{+}$-recipient mice (Supplementary Fig. 3a,b). Cell fate of donor cells, distinguished from recipient by expression of GFP and congenic CD45, was analysed by flow cytometry 2 and 4 days after transfer in BM and spleen. When analysed by GFP and Ly6C expression, transferred Ly6 $\mathrm{C}^{\text {hi }}$ monocytes progressively and uniformly switched to a Ly6 $\mathrm{C}^{\mathrm{lo}}$ monocyte phenotype displaying upregulation of GFP and downregulation of Ly6C (Fig. 1d, Supplementary Fig. 3c). An extended marker analysis demonstrated more complex phenotypic changes involving the progressive acquisition of CD11c and CD43 while maintaining low expression levels of major histocompatibility complex (MHC)-II, consistent with conversion into Ly6 $\mathrm{C}^{\mathrm{lo}}$ monocytes. These changes occurred over a period of 4 days and were observed in BM and spleen (Fig. 1d). Thus, an extended phenotypic analysis confirms conversion of Ly6C hi monocytes into Ly6C ${ }^{\text {lo }}$ monocytes.

Notch2 regulates $\mathrm{Ly}_{6} \mathrm{C}^{\mathrm{lo}}$ monocytes in vivo. To evaluate a potential role of Notch signalling in monocyte subset regulation we sorted Ly6 $\mathrm{C}^{\mathrm{hi}}$ and Ly6 $\mathrm{C}^{\text {lo }}$ monocytes from the BM and analysed Notch-related gene-expression patterns. Compared with Ly6 $\mathrm{C}^{\text {hi }}$ monocytes, Ly6 $\mathrm{C}^{\text {lo }}$ monocytes had lower expression of Notch 1 but comparable Notch 2 expression in messenger RNA and protein (Fig. 2a,e,f). Furthermore, Notch-regulated genes, Hey2 
and Hes1, were markedly induced in Ly6C ${ }^{\text {lo }}$ monocytes, indicating recent or on-going activation of Notch signalling in this subset (Fig. 2a) ${ }^{3,30}$. We next wanted to confirm these findings on corresponding human monocyte subsets. Analysis of the human $\mathrm{CD} 6^{+}$monocytes, which are considered equivalents of mouse Ly6 $\mathrm{C}^{\text {lo }}$ monocytes, revealed higher expression of HES1 compared with the classical CD14 ${ }^{+}$monocytes (Fig. 2b).

We next asked whether Notch deficiency influences monocyte subpopulations. To generate mice with conditional deletions of Notch receptors in monocytes we crossed mice bearing floxed a

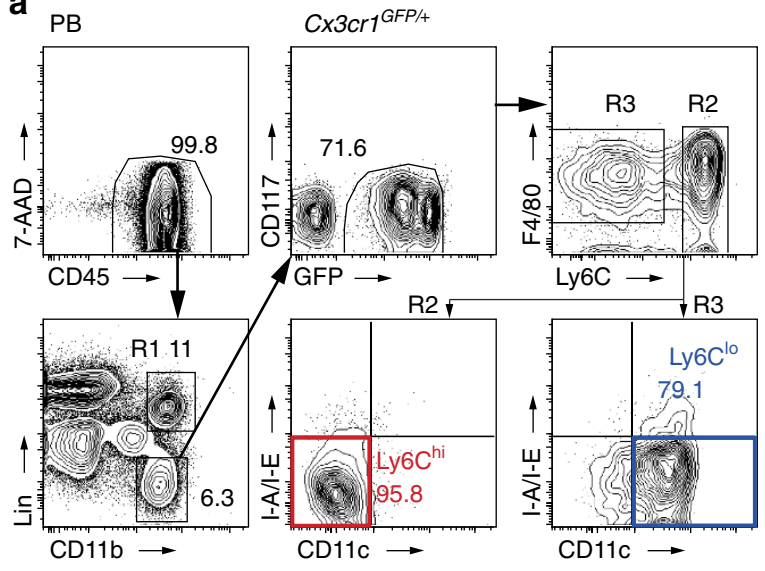

b $\quad \mathrm{FSC} / \mathrm{SSC}$.

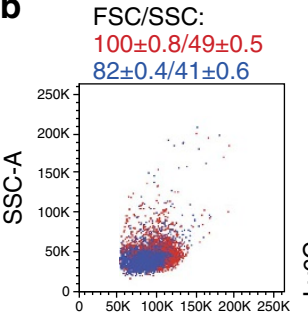

FSC-A

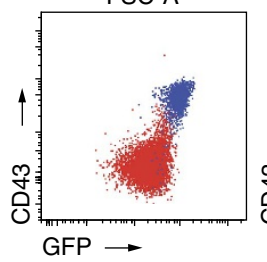

C

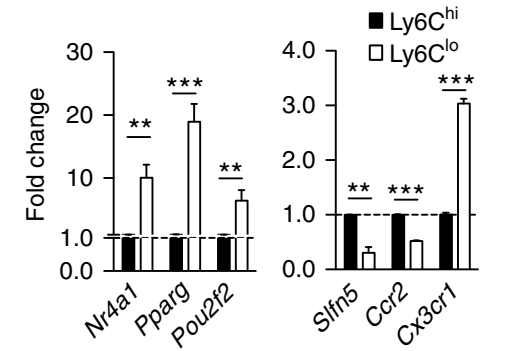

d
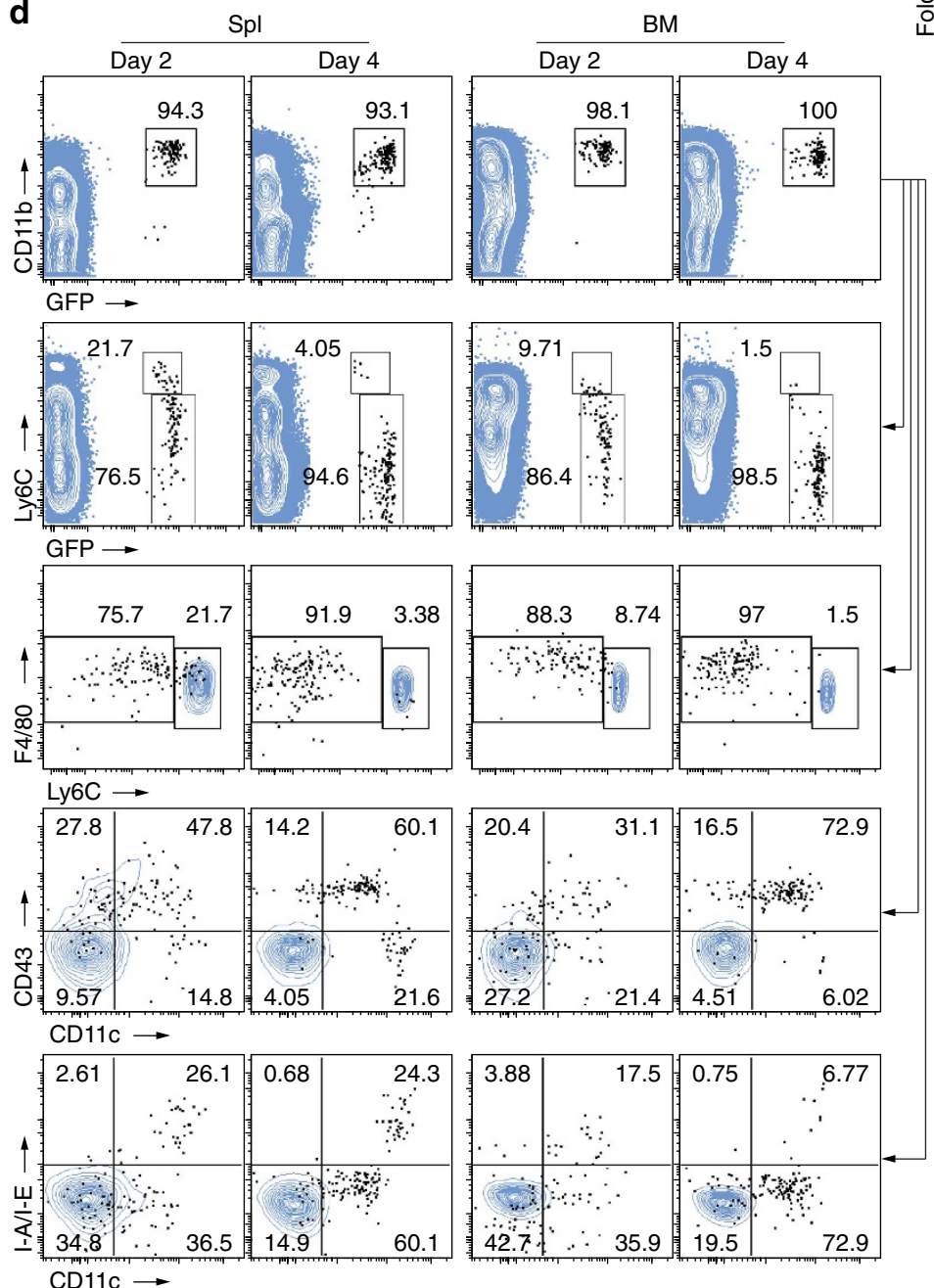

- CD $45.2^{+} \mathrm{CD} 11 \mathrm{~b}^{+} \mathrm{GFP} \mathrm{P}^{+}$ CD $45.1^{+}$CD $11 b^{+}$Ly6C $C^{\text {hi }} 4 / 80^{\text {lo }}$ 
alleles of Notch1, Notch2 or Notch1/Notch2 (refs 17,31) with a myeloid specific Cre-recombinase strain, LysM Cre (ref. 32). Strains were also back-crossed onto the C $x 3 c r 1^{G F P /+}$ reporter strain (Supplementary Table 2). This targeting strategy was characterized in detail. LysM reporter analysis in LysM-eGFP mice confirmed low $L y s M$ promoter activity in progenitor populations, but high promoter activity in Ly6 $\mathrm{C}^{\text {hi }}$ and Ly6Clo monocytes (Supplementary Fig. $4 \mathrm{a})^{33}$. In addition, crossing the Lys $M^{C r e}$ strain to a Cre-dependent YFP reporter strain revealed selective mature myeloid targeting, which was partial in $\mathrm{Ly}_{6} \mathrm{C}^{\text {hi }}$ monocytes, and more efficient for Ly6C ${ }^{\text {lo }}$ monocytes and granulocytes (Fig. 2c, Supplementary Fig. 4b), confirming previous reports 34 .

Mice with conditional deletion of Notch2 $\left(G F P^{+} N 2^{\Delta M y}\right)$ had significantly reduced absolute and relative numbers of Ly6 $\mathrm{C}^{\text {lo }}$ monocytes in BM, PB and spleen (Fig. 2d, Supplementary Fig. 5a). The defect was only observed in Ly6 $\mathrm{C}^{\text {lo }}$ monocytes, since numbers of Ly6C hi monocytes, monocyte progenitor populations and neutrophils were unaffected, and occurred independent of the Gfp reporter allele or the Cre deleter allele (Fig. $2 \mathrm{~d}$ and Supplementary Fig. 5b-d). In contrast, mice with conditional deletion of Notch1 showed no alteration in monocyte subsets (Supplementary Fig. 5e), while combined deletion of Notch1/Notch 2 phenocopied the single Notch2 mutants (Supplementary Fig. 5f). Altogether, these results demonstrate that monocyte Notch2 controls Ly6Clo monocyte numbers, suggesting a role in monocyte cell fate regulation.

To further investigate the selective reduction of $\mathrm{Ly}_{6} \mathrm{C}^{\mathrm{lo}}$ monocytes we next characterized Notch2 receptor expression by flow cytometry in control mice and conditional mutants. Notch2 was robustly expressed in MDP, cMoP, Ly6Chi and Ly6Clo monocytes in control mice (Fig. 2e,f). In $G F P^{+} N 2^{\Delta M y}$ mice, Notch2 expression was not affected in MDPs and cMoP, but substantially reduced in Ly6 $\mathrm{C}^{\text {hi }}$ monocytes, consistent with partial Cre expression and activity in this population (Fig. 2e,f, Supplementary Fig. 4c and (ref. 34)). However, although Cremediated targeting is more effective in Ly6 $\mathrm{C}^{\mathrm{lo}}$ monocytes (Fig. $2 \mathrm{c}$, Supplementary Fig. 4b) ${ }^{34}$, most remaining Ly6C $\mathrm{C}^{\text {lo }}$ monocytes in Notch 2 mutant mice retained normal Notch2 receptor expression, due to low expression of Cre (Fig. 2e,f and Supplementary Fig. 4c). This suggests that the remaining Ly6 $\mathrm{C}^{\text {lo }}$ monocytes in these mice develop from Notch2-expressing (Cre-negative) Ly6 $\mathrm{C}^{\text {hi }}$ monocytes (Supplementary Fig. 4c). Consistent with this idea, increasing the efficiency of Notch2 deletion in Ly6 $\mathrm{C}^{\text {hi }}$ monocytes, by using Notch $2^{f / f}$ mice carrying two alleles of $L y s M^{C r e}$, increased the rate of Notch2 deletion in Ly6 $\mathrm{C}^{\text {hi }}$ monocytes but also lead to more strongly reduced levels of Ly6C ${ }^{\text {lo }}$ monocytes (Fig. $2 \mathrm{~g}, \mathrm{~h}$ ), while Ly6 $\mathrm{C}^{\mathrm{hi}}$ monocyte levels remained normal (Fig. $2 \mathrm{~g}$ ). Thus, loss of Notch2 at the level of Ly6C ${ }^{\text {hi }}$ monocytes is compatible with generation of Ly $6 \mathrm{C}^{\mathrm{hi}}$ monocytes, but incompatible with generation of Ly6C $\mathrm{C}^{\mathrm{lo}}$ monocytes. Indeed, plotting Notch2 receptor levels on Ly6C monocytes against the frequency of Ly6 $\mathrm{C}^{\text {lo }}$ monocytes revealed that loss of Notch2 on Ly6 $\mathrm{C}^{\mathrm{hi}}$ monocytes correlated strongly with loss of $\mathrm{Ly}^{\mathrm{lo}}$ monocytes (Fig. 2h). This also suggested that the fate of $\mathrm{Ly}_{6} \mathrm{C}^{\mathrm{lo}}$ monocytes is linked to Ly6C hi monocytes through Notch2.

We next wanted to test the effects of a more selective deletion of Notch 2 within the Ly6 $\mathrm{C}^{\text {lo }}$ population. To this end we used a conditional CD11c-Cre transgenic approach to delete Notch2 at early stages of Ly6C $\mathrm{C}^{\text {lo }}$ monocyte development, since CD11c is selectively upregulated in $\mathrm{Ly} \mathrm{C}^{\text {lo }}$ monocytes at early stages of conversion (Fig. 1d, Fig. 5a). Loss of Notch2 partially reduced the number of Ly6C $\mathrm{C}^{\text {lo }}$ monocytes without affecting Ly6C ${ }^{\text {hi }}$ monocytes (Fig. 2i, Supplementary Fig. 4d). Altogether, this demonstrates a requirement for Notch2 in the generation and maintenance of Ly6C ${ }^{\text {lo }}$ monocytes.

Physiologic consequences of monocyte Notch2 deficiency. To study the consequences of Notch2 loss of function for monocyte patrolling behaviour in more detail we performed intravital microscopy of the cremaster muscle ${ }^{8,35}$. Under steady-state conditions, the number of rolling and adherent Ly6C ${ }^{\text {lo }}$ monocytes was greatly reduced in $\mathrm{GFP}^{+} \mathrm{N} 2^{4 M y}$ mice, which confirmed the results obtained by flow cytometry (Fig. 3a,b). Furthermore, while TNF- $\alpha$ treatment increased the rolling and adherence of Ly6C $\mathrm{C}^{\mathrm{lo}}$ monocytes in control mice, this response was blunted in $G F P^{+} N 2^{\Delta M y}$ mice (Fig. 3a,b), demonstrating a profound impairment of the Ly6C lo monocyte subpopulation with Notch 2 loss of function. This result also ruled out the possibility that mutant Ly6 $\mathrm{C}^{\mathrm{lo}}$ monocytes preferentially localize to blood vessel walls. In addition, the reduction of Ly6 $\mathrm{C}^{\mathrm{lo}}$ monocytes in Notch 2 mutant mice was accompanied by accumulation of an atypical cell population expressing high levels of MHC-II and CCR2 but low levels of CD11c, CD43 and CD11a (Fig. 3c-e), a phenotype not resembling a previously described $\mathrm{MHC}-\mathrm{II}^{+}$monocyte population ${ }^{36}$.

Notch2 regulates Ly6 $\mathrm{C}^{\text {hi }}$ monocyte conversion in vivo. Ly6C $\mathrm{C}^{\mathrm{lo}}$ monocyte deficiency could be due to increased cell death, as was shown in mice deficient for the transcription factor Nr4al, which controls Ly6C lo monocyte numbers in part by regulating monocyte apoptosis ${ }^{13}$. We, therefore, tested the hypothesis that Notch2 regulates monocyte survival. However, neither the fraction of apoptotic cells, nor the fraction of dead cells was altered in each of the monocytes subsets in BM, PB or spleen in conditional Notch 2 mutants (Fig. 4a,b).

To address the question whether conversion of $\mathrm{Ly}_{6 \mathrm{C}} \mathrm{hi}$ monocytes depends on Notch 2 we isolated Ly6C ${ }^{\text {hi }}$ monocytes from $\mathrm{C} x 3 \mathrm{cr} 1^{\mathrm{GFP} /+}$ control mice or conditional Notch2 mutants and analysed the cell fate after adoptive transfer into CD45.1 ${ }^{+}$ congenic wild-type recipients. Four days after transfer the majority of recovered donor cells from control donors had converted into Ly6C $\mathrm{C}^{\text {lo }}$ monocytes, while few remained Ly6C ${ }^{\text {hi }}$ monocytes. After transfer of cells from conditional mutants, however, the fraction of donor cells that had converted into

\footnotetext{
Figure 1 | Identification of monocyte subsets and lineage relationships. (a) Monocyte subpopulation analysis strategy in PB of Cx3cr ${ }^{G F P /}+$ mice. Initially cells were identified based on FSC and SSC characteristics. After exclusion of doublets (on the basis of SSC-W, SSC-A) Lin ${ }^{-}$CD11b ${ }^{+}$cells were gated from

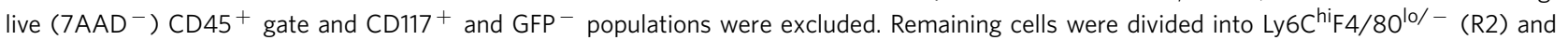
Ly6Clo/ - F4/80 lo (R3) subsets. Ly6C hi monocytes were defined from R2 as CD11c- MHC-II lo/ - (red) and Ly6Clo monocytes from R3-as CD11 ${ }^{\text {lo }}$

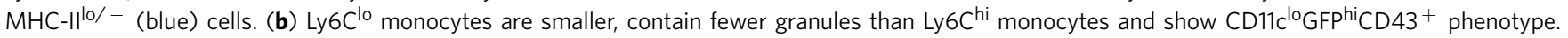
Numbers are mean \pm s.e.m. (c) Quantitative reverse transcription-PCR analysis performed in sorted monocyte subsets from BM of $C \times 3 c r 16 F P /+$ mice. Change relative to expression in Ly6Chi cells is shown $(n=3 / 6)$. Error bars represent s.e.m. ${ }^{\star} P<0.05,{ }^{\star \star} P<0.01$, ${ }^{\star \star \star} P<0.001$; Student's $t$-test. (d) Dynamics of Ly6Clo monocyte development. BM CD45.2 ${ }^{+} \mathrm{CD} 11 \mathrm{~b}+\mathrm{GFP}+$ Ly6Chi monocytes were transferred into CD45.1 ${ }^{+}$recipients and their conversion into Ly $6 \mathrm{C}^{10}$ monocytes were followed in vivo. Flow cytometry analysis of recipient spleen and BM is depicted. Transferred cells are black and for

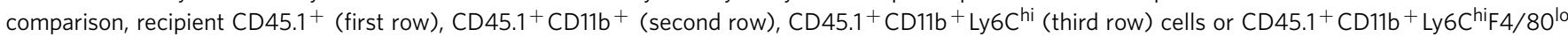
monocytes (fourth and fifth rows) are shown in blue (representative of two experiments).
} 
a

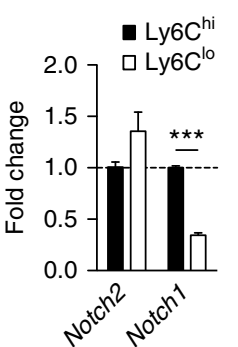

b

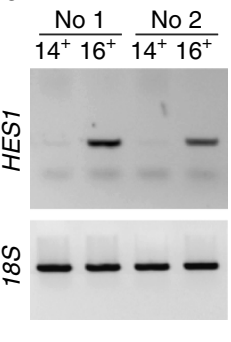

c

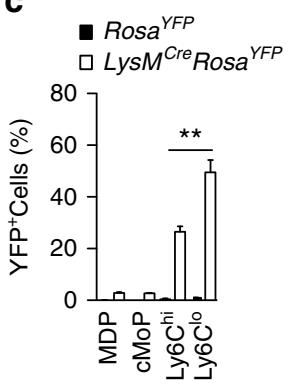

e

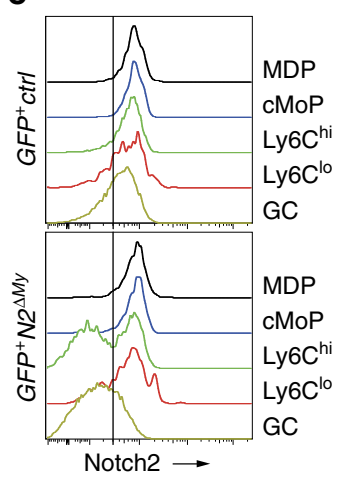

d - $G F P^{+} c t r l$ $\square$ GFP $N 2^{\triangle M y}$
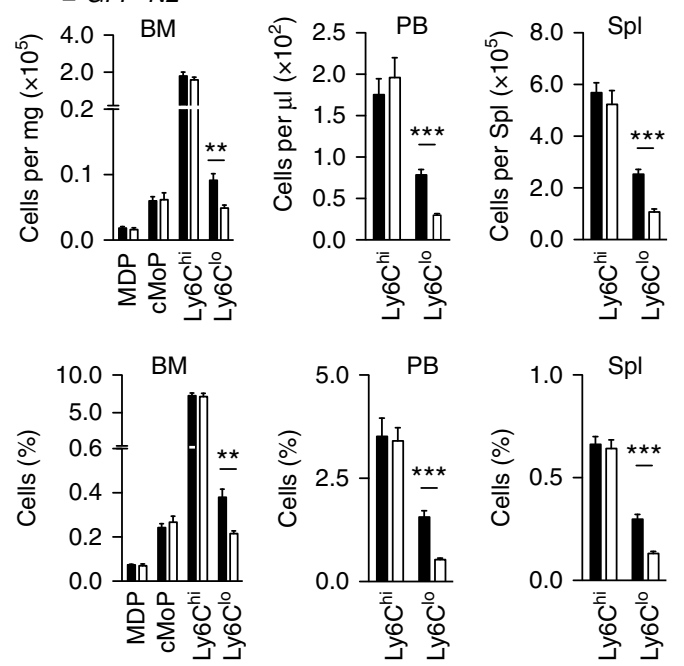

g

- $G F P^{+} c t r l$

$\square$ GFP ${ }^{+}$Cre/Cre
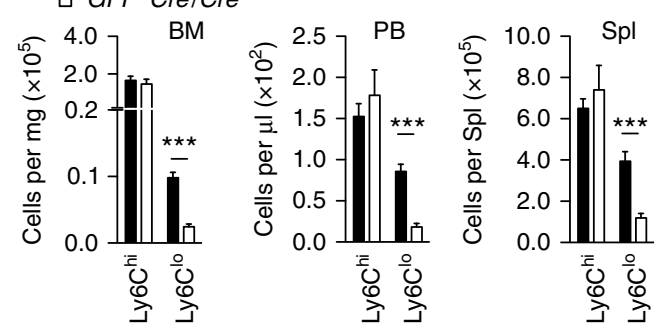

h
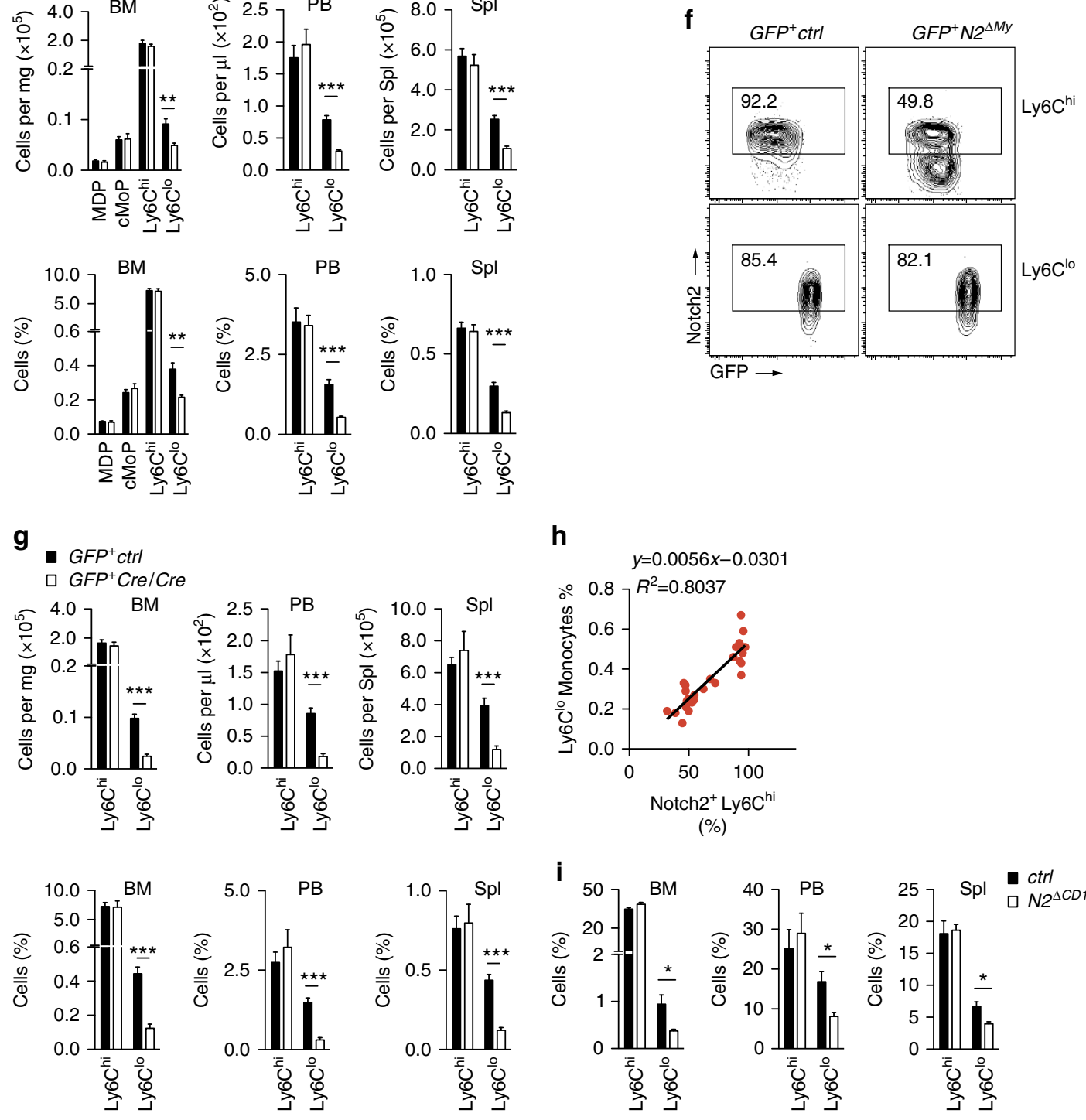

(\%)
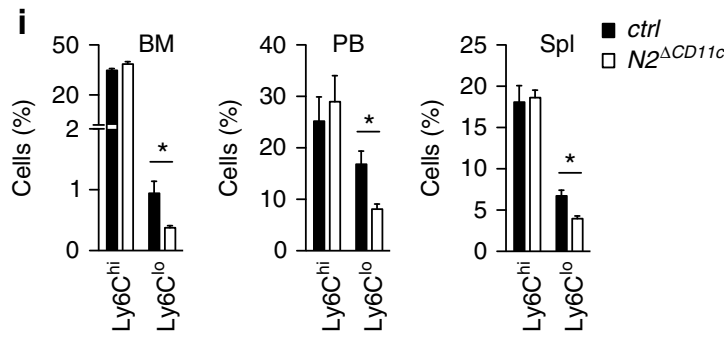

Figure 2 | Conditional deletion of Notch2 impairs Ly6 $\mathbf{C}^{\mathbf{l o}}$ monocyte development. (a) Quantitative reverse transcription-PCR analysis in sorted monocyte subsets from BM of $\mathrm{C} \times 3 \mathrm{cr} 7^{\mathrm{GFP} /+}$ mice; $\left(n=6\right.$, pooled from three experiments). (b) HES1 expression in human $\mathrm{CD} 14^{+}$(classical) or CD16 ${ }^{+}$(non-classical) monocytes from two donors. (c) Quantification of YFP ${ }^{+}$cells in myeloid cells from LysM ${ }^{\text {Cre }}$ Rosa ${ }^{\text {YFP }}$ mice as a hallmark of Cre activity. Data are pooled from two experiments with three mice in each group. (d) Flow cytometry of myeloid cell subpopulations in mice with conditional deletion of Notch2. Absolute number of cells per mg BM, per $\mu$ l blood or per spleen is shown (top). Relative frequency of each subpopulation from live cell gate is shown (bottom). Data are pooled from three experiments with $11 / 8$ mice in each group. (e) Notch2 expression in myeloid cell subpopulations from BM of GFP ${ }^{+}$Notch ${ }^{4 M y}$ mice. (f) Notch2 expression in Ly6Chi and Ly6C $\mathrm{C}^{\mathrm{lo}}$ monocyte subpopulations isolated from BM. Littermate controls are shown for comparison. (g) Quantification of monocyte subpopulations in mice with conditional deletion of Notch2 and expressing two alleles of Lys $M^{C r e}$. Absolute number of cells per mg BM, per $\mu$ l blood or per spleen is shown (top). Relative frequency of each subpopulation from live cell gate is shown (bottom). Data are pooled from three experiments with $12 / 7$ mice in each group. (h) Correlation of Notch2 ${ }^{+}$Ly $6 C^{\text {hi }}$ monocyte frequency with frequency of Ly6C ${ }^{l o}$ monocytes. Frequency of Notch2 ${ }^{+}$Ly $6 C^{h i}$ monocytes shows strong positive correlation with Ly6 $6 \mathrm{C}^{\mathrm{lo}}$ monocyte numbers $(n=28)$. (i) Quantification of monocyte subpopulations in Notch $2^{\Delta C D 11 c}$ mice. Data are pooled from two experiments with four mice in each group. $(\mathbf{a}, \mathbf{c}, \mathbf{d}, \mathbf{g}, \mathbf{i}){ }^{\star} P<0.05$, ${ }^{\star \star} P<0.01,{ }^{\star \star \star} P<0.001$; Student's $t$-test. Error bars represent s.e.m. 
a

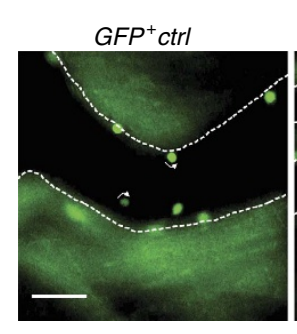

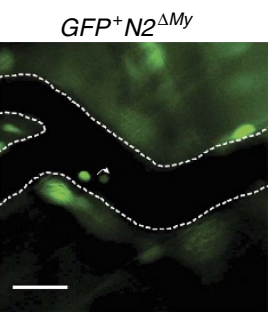

b

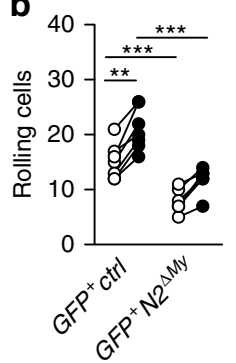

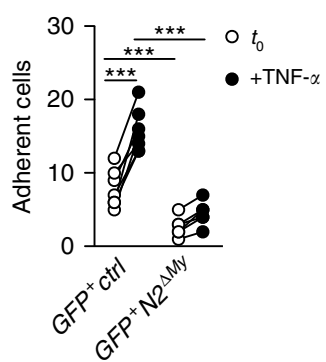

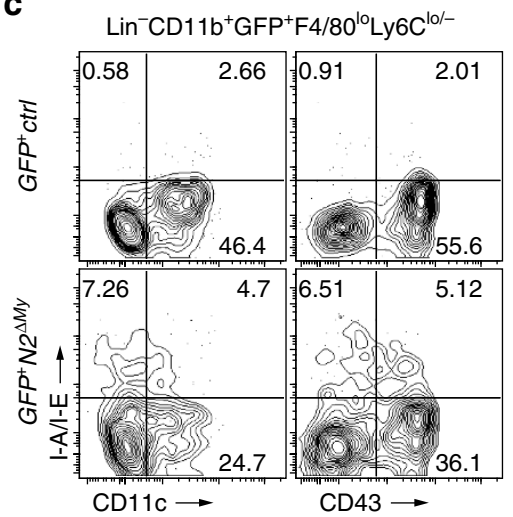

d

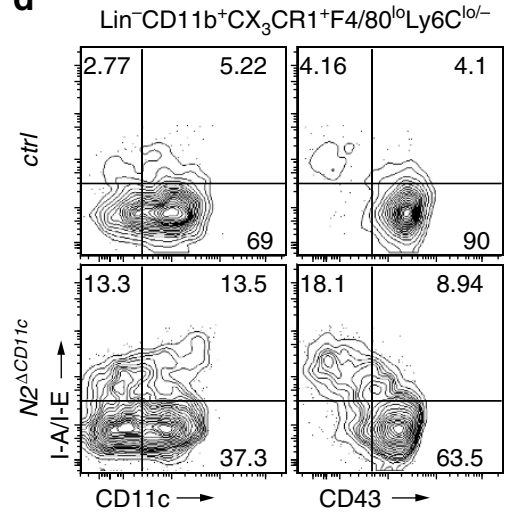

e - Ly6C $^{10}$ Atypical cells

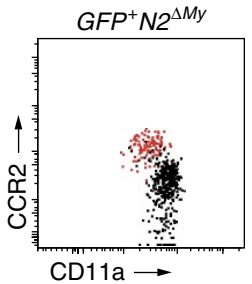

Figure 3 | Ly6 $\mathbf{C}^{\mathbf{l 0}}$ monocytes show quantitative and phenotypical defects. (a) Intravital microscopy image showing GFP ${ }^{+}$cells in microcirculation of cremaster. Scale bar, $50 \mu \mathrm{m}$. (b) Quantification of adherent (left) or rolling (right) monocytes per field of view in cremaster blood vessels at $t=0$ or 60 min after infusion of TNF- $\alpha$. Data are pooled from two experiments $(n=6 / 7)$. ${ }^{\star \star \star} P<0.001$; two-way ANOVA with Bonferroni's post-test. (c,d) GFP ${ }^{+}$Notch $2^{4 M y}$ (c) and Notch2 ${ }^{4 C D 11 c}$ (d) mice develop an atypical CD11c ${ }^{-} \mathrm{CD} 43^{-} \mathrm{MHC}-\mathrm{II}^{+} \mathrm{Ly}_{6 \mathrm{C}}{ }^{\mathrm{lo}}$ population. Representative plots from $\mathrm{Lin}^{-} \mathrm{CD} 11 \mathrm{~b}+\mathrm{GFP}+\mathrm{F} 4 /$ $80^{\mathrm{lo}} \mathrm{Ly} 6 \mathrm{C}^{\mathrm{lo} /}-$ or $\mathrm{Lin}^{-} \mathrm{CD} 11 \mathrm{~b}+\mathrm{CX}_{3} \mathrm{CR} 1^{+} \mathrm{F} 4 / 80^{\mathrm{lo}} \mathrm{Ly} 6 \mathrm{C}^{\mathrm{lo} /}-$ parental gates, respectively. (e) Representative flow cytometry plot showing CCR2 and CD11a expression in Ly6Clo monocytes or atypical cells.

Ly6C ${ }^{\text {lo }}$ monocytes was strongly reduced, while the fraction of recovered Ly6C hi monocytes was significantly increased (Fig. 4c,d, Supplementary Fig. 6). Similar results were obtained in experiments when peripheral Ly6 $\mathrm{C}^{\text {hi }}$ monocytes retrieved from $\mathrm{PB}$ and spleen were used for transfer (Supplementary Fig. 7), which also ruled out development of Ly6C ${ }^{l o}$ monocytes from contaminating BM precursors. Thus, Ly6 $\mathrm{C}^{\text {hi }}$ monocytes deficient for Notch2 show impaired conversion into Ly6 $\mathrm{C}^{\text {lo }}$ monocytes, demonstrating regulation of monocyte cell fate by Notch2.

Dll1-Notch2 axis controls monocyte conversion in vitro. Notch receptors are differentially engaged by Notch ligands, and Notch2 is a preferred target of DLL1 (ref. 24). To define the Notch signalling components regulating monocyte conversion, and to provide proof-of-principle that Notch activation is sufficient to regulate this process, we established an in vitro culture system to mimic the initial steps during monocyte conversion under defined conditions. We sorted Ly6 $\mathrm{C}^{\text {hi }}$ monocytes from $\mathrm{BM}$ of $C \times 3 \mathrm{cr} 1^{G F P /+}$ mice (Fig. 5a day 0) and cultured them in the presence of immobilized recombinant DLL1 protein, or control conditions (Fig. 5a and Supplementary Fig. 8). In culture, downregulation of Ly6C and upregulation of GFP occurred in all conditions over time, while cells remained uniformly CD115 (Fig. 5a, Supplementary Fig. 8b). However, conversion of Ly6C ${ }^{\text {hi }}$ into Ly6 $\mathrm{C}^{\mathrm{lo}}$ monocyte-like cells, detected by upregulation of CD43 and CD11c in MHC-II ${ }^{\mathrm{lo} /}$ - cells, occurred to a significantly greater extent on DLL1 than in control cultures (Fig. 5a,b). Furthermore, in gene expression profiling, cells cultured on DLL1 showed significantly higher levels of Nr4a1 and Pou2f2 and significantly lower levels of Slfn 5 compared with control culture (Fig. 5c), similar to a Ly6C $\mathrm{C}^{\text {lo }}$ monocyte phenotype ${ }^{3}$.
The effect was specific for DLL1, since culture of Ly6C ${ }^{\text {hi }}$ monocytes on another Notch ligand, JAG1, was much less effective in generating Ly6C $\mathrm{C}^{\text {lo }}$ monocyte-like cells, as were control conditions (Fig. 5d,e). Furthermore, DLL1-induced monocyte conversion was impaired by incubation with a $\gamma$-secretase inhibitor, N-(N-(3,5-difluorophenacetyl)-L-alanyl)-S-phenylglycine t-butyl ester (DAPT), which blocks the generation of the active intracellular Notch domain ${ }^{37}$, thus indicating that DLL1-induced Notch receptor cleavage is required for this process (Fig. 6a,b). Importantly, DLL1-induced monocyte conversion was also severely impaired in Ly6 $\mathrm{C}^{\text {hi }}$ monocytes from Notch2 conditional mutants when compared with controls (Fig. 6c,d). These results demonstrate that a specific Dll1-Notch2 signalling axis controls conversion of Ly6 $\mathrm{C}^{\text {hi }}$ monocytes into Ly6C ${ }^{\text {lo }}$ monocyte-like cells.

Dll1 is expressed in distinct endothelial niches. We wanted to corroborate the specific function of Dll1 for Ly6C ${ }^{\text {lo }}$ monocyte development in vivo. In the adult mouse, Dll1 is selectively expressed in vascular endothelium of arteries, but not veins or capillaries, and in EC in the MZ of the spleen ${ }^{24,37}$. We first characterized in more detail Dll1 expression in the two principle haematopoietic compartments, BM and spleen, using genetic reporter mice or immunostaining. In $\mathrm{Dll} 1^{+}$hacZ reporter mice, in which one allele of Dll1 has been replaced by lac $Z$, specific reporter staining was observed in the splenic MZ, but not in the central artery of the splenic follicle (Fig. 7a). Immunofluorescence staining against CD31 and DLL1 and confocal microscopy revealed DLL1 expression in EC of the MZ and confirmed its absence in the central artery of the follicle (Fig. 7b). Interestingly, DLL1 staining in $C x 3 c r 1^{G F P /+}$ reporter mice demonstrated a 

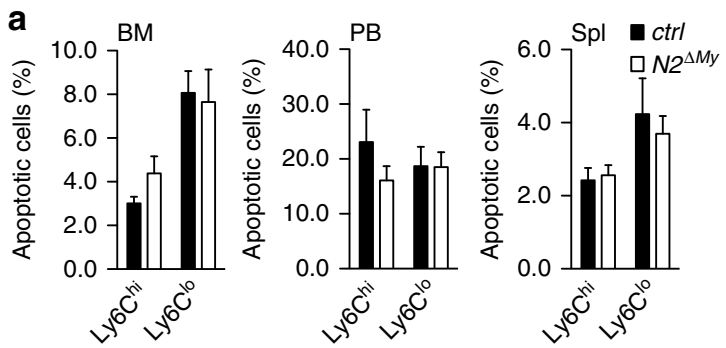
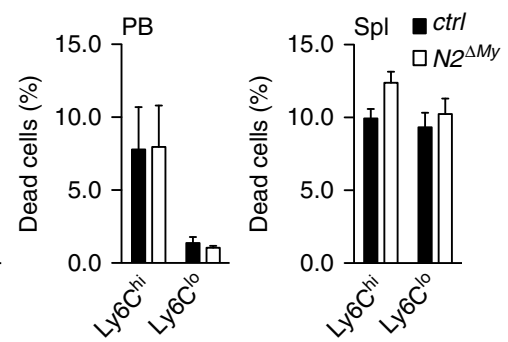

C
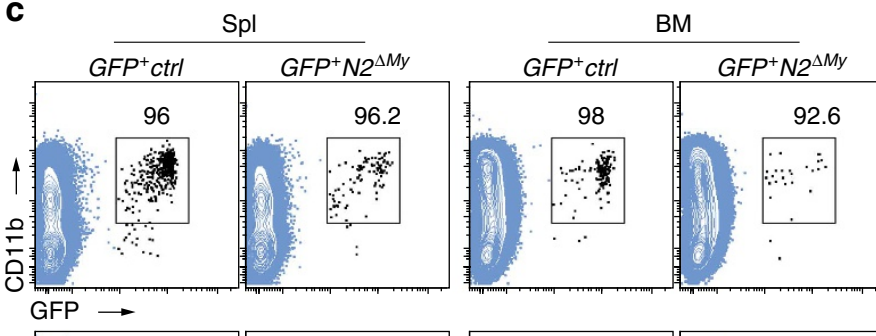

- Donor CD45.2 GFP $^{+}$

Recipient CD45.1 $1^{+}$
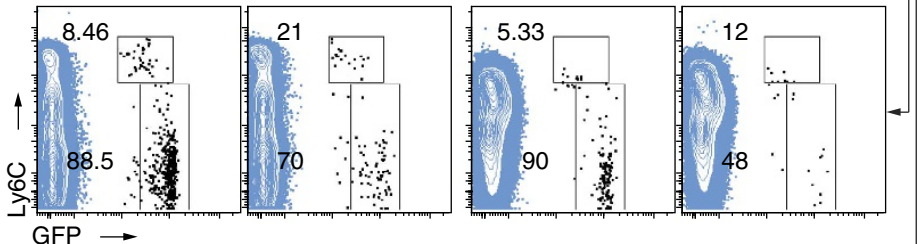

- CD $45.2^{+} \mathrm{CD} 11 \mathrm{~b}^{+} \mathrm{GFP}$

- CD $45.1^{+} \mathrm{CD} 11 \mathrm{~b}^{+}$
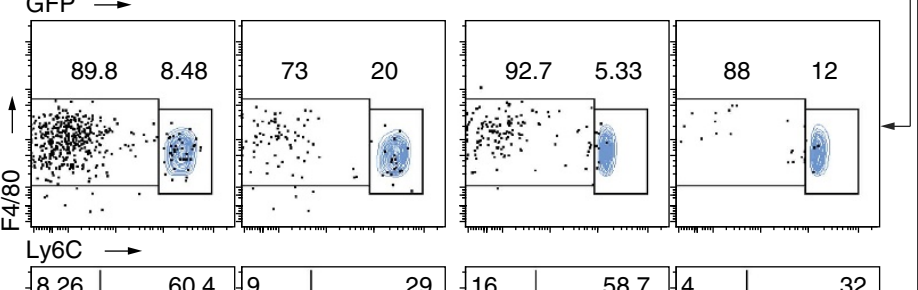

- CD45.2 $2^{+} \mathrm{CD} 11 \mathrm{~b}^{+} \mathrm{GFP} \mathrm{P}^{+}$

- CD $45.1^{+} C D 11 b^{+}$Ly6C
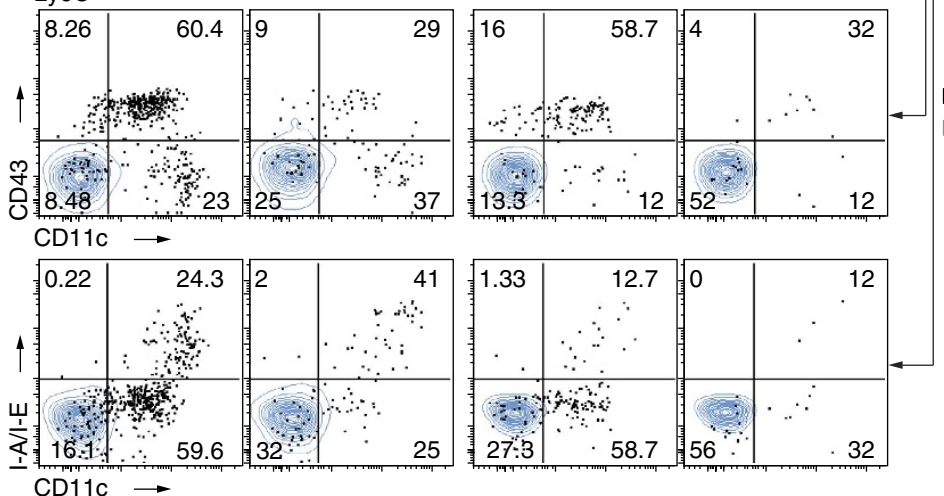

- CD $45.2^{+} C D 11 b^{+} G F P^{+}$
CD $45.1^{+} C D 11 b^{+} L y 6 C^{h i} F 4 / 80^{l o}$

d $\quad \mathrm{CD} 45.2^{+} \mathrm{CD} 11 \mathrm{~b}^{+} \mathrm{GFP}{ }^{+}$:

- $G F P^{+} \mathrm{ctrl}$

$\square G F P^{+} N 2^{\Delta M y}$

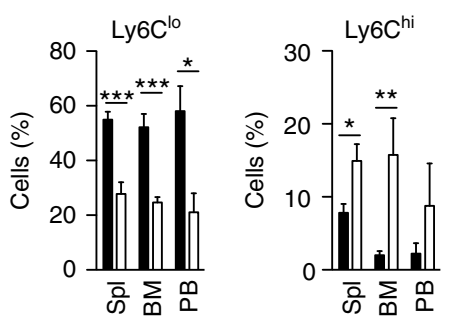

Figure 4 | Notch2-deficient Ly6 $\mathbf{C}^{\text {hi }}$ monocytes show impaired conversion potential in vivo. Flow-cytometry based quantification of the fraction of (a) apoptotic cells (Annexin $\mathrm{V}^{+} 7-\mathrm{AAD}^{-}$) or (b) dead cells $\left(7-\mathrm{AAD}^{+}\right)$in monocyte subsets. Data are pooled from three-independent experiments $(n=8 / 12)$. (c) Flow cytometry four days after adoptive transfer of BM Ly6Chi monocytes from control or Notch2-deficient CD45.2 ${ }^{+} \mathrm{GFP}+$ donors into CD45.1 ${ }^{+}$congenic recipients. Transferred cells are shown in black and for comparison, recipient CD45.1 ${ }^{+}$(first row), CD45.1 ${ }^{+}$CD11b ${ }^{+}($second row), CD 45.1 ${ }^{+}$CD11b ${ }^{+}$Ly6Chi (third row) cells or CD45.1 ${ }^{+}$CD11b ${ }^{+}$Ly6ChiF4/80lo monocytes (fourth and fifth rows) are depicted in blue. (d) Frequency of donor-derived Ly6 $\mathrm{C}^{\mathrm{lo}}$ monocytes (left) or Ly6Chi monocytes (right) in the CD11b $+\mathrm{GFP}+\mathrm{CD} 45.2^{+}$gate after adoptive transfer of Ly6Chi monocytes. Data are pooled from four-independent experiments $(n=5 / 7)$. (a,b, d $){ }^{\star} P<0.05,{ }^{\star \star} P<0.01,{ }^{\star \star \star} P<0.001$; Student's $t$-test. Error bars represent s.e.m.

close spatial relationship between DLL1 expression and GFP ${ }^{+}$ cell populations in the MZ, suggesting a potential niche function (Fig. 7c). Co-staining with Ly6C or CD43 identified both monocyte subsets within the $\mathrm{CD} 31^{+}$and $\mathrm{DLL1}^{+} \mathrm{MZ}$ area, while large $\mathrm{GFP}^{+}$macrophages reside in the borders of the $\mathrm{MZ}$ (Fig. 7d,e and Supplementary Fig. 9a,b). Furthermore, specific Dll1-reporter staining in Dll1 +/lacZ mice was also observed in the
$\mathrm{BM}$, which appeared in a reticular pattern in the diaphysal area of the BM cavity, suggesting a vascular pattern (Fig. 7f). More importantly, the defect in Ly6C ${ }^{\text {lo }}$ monocytes observed in Notch2 conditional mutants was recapitulated in haploinsufficient Dll1 mutant mice (Fig. $7 \mathrm{~g}$ ). This demonstrates a critical role for Dll1 in the regulation of $\mathrm{Ly}^{\mathrm{C}} \mathrm{C}^{\mathrm{lo}}$ monocytes in vivo, and emphasizes the importance of a Dll1-Notch2 axis in the control of Ly6C $\mathrm{C}^{\mathrm{lo}}$ 
a

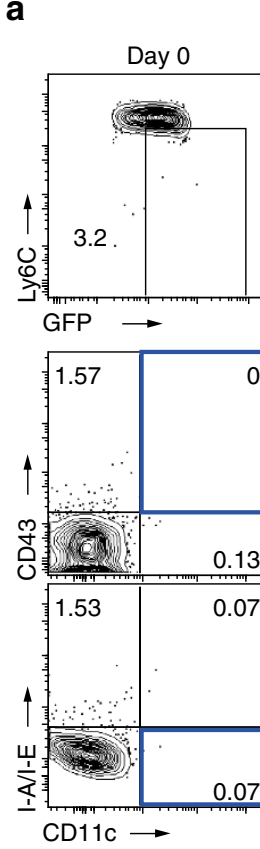

b

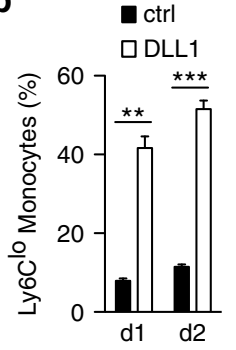

Day 1
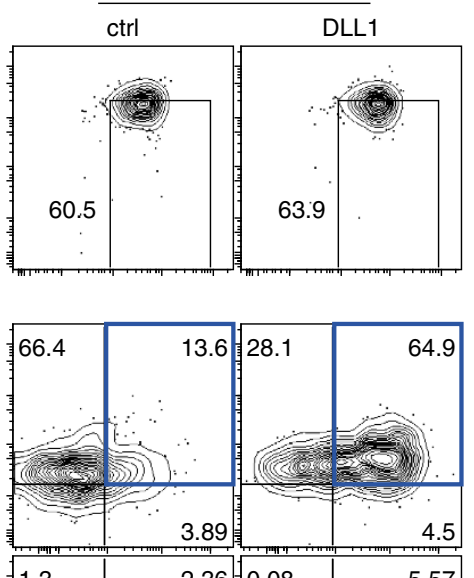

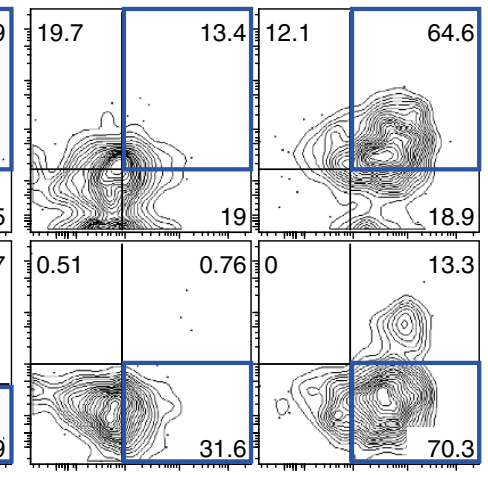

15.2
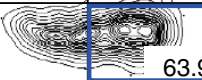
63.9

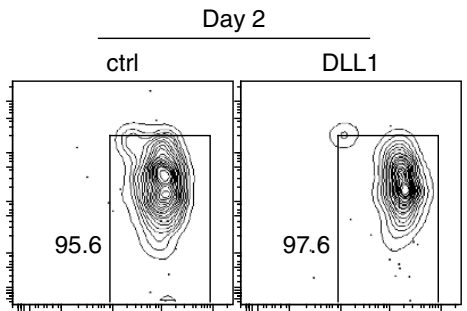

C
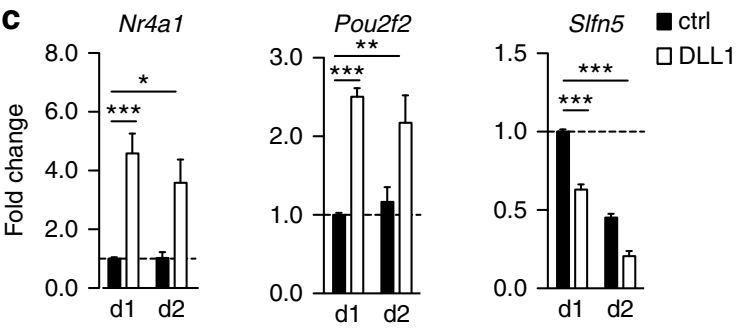

d CD11b ${ }^{+}$GFP $^{+}$Ly6C $C^{\mathrm{lo} /-}$ gate:

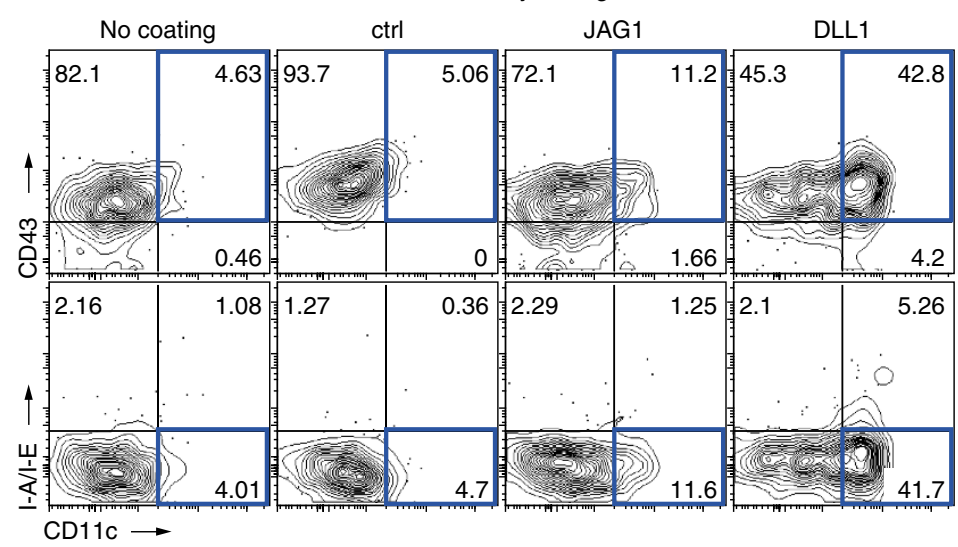

e

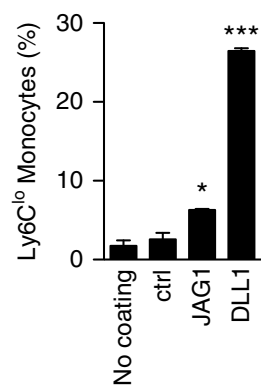

Figure 5 | Notch ligand DLL1 mediates monocyte conversion in vitro. $(\mathbf{a}, \mathbf{b})$ Kinetics of monocyte conversion in the presence of Notch ligand DLL1 in vitro.

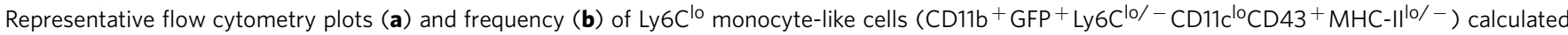
from live $\mathrm{CD}_{11 \mathrm{~b}}{ }^{+} \mathrm{GFP}+$ cells are shown $(n=3)$. (c) Gene expression analysis in in vitro cultures from sorted BM Ly6Chi monocytes. Change relative to expression of genes in control cultures is shown $(n=6)$. (d,e) DLL1 specifically induces conversion in vitro. Representative flow cytometry plot (d) and frequency (e) of Ly6 $\mathrm{C}^{10}$ monocyte-like cells (similar to Fig. 5a,b) are shown after culture of Ly6Chi monocytes alone or in the presence of control, JAG1 or DLL1 ligands. $(\mathbf{b}, \mathbf{c}, \mathbf{e}){ }^{\star} P<0.05,{ }^{\star \star} P<0.01,{ }^{\star \star \star} P<0.001$; one-way ANOVA with Bonferroni's multiple comparison test. Error bars represent s.e.m.

monocyte development. Furthermore, these observations also suggest that distinct Dll1 ${ }^{+}$EC might form specialized niches for the generation of Ly6C $\mathrm{C}^{\text {lo }}$ monocytes.

Endothelial Dll1 controls Ly6 $\mathrm{C}^{\text {lo }}$ monocyte development. To test the hypothesis that endothelial Dll1 regulates Ly6 $\mathrm{C}^{\text {lo }}$ monocyte development we employed an endothelial-specific and inducible deletion strategy using the Cdh5(PAC)-CreERT2 strain, which shows EC-specific Cre activity in peripheral vessels and the BM cavity $^{16,38}$. We first confirmed pan-endothelial, but EC-specific, targeting by generating Cre-dependent lacZ-reporter mice $\left(\right.$ lac $\left.Z^{i E C}\right)$, which demonstrated inducible EC staining in arteries, veins and capillaries after tamoxifen treatment, but also 
a
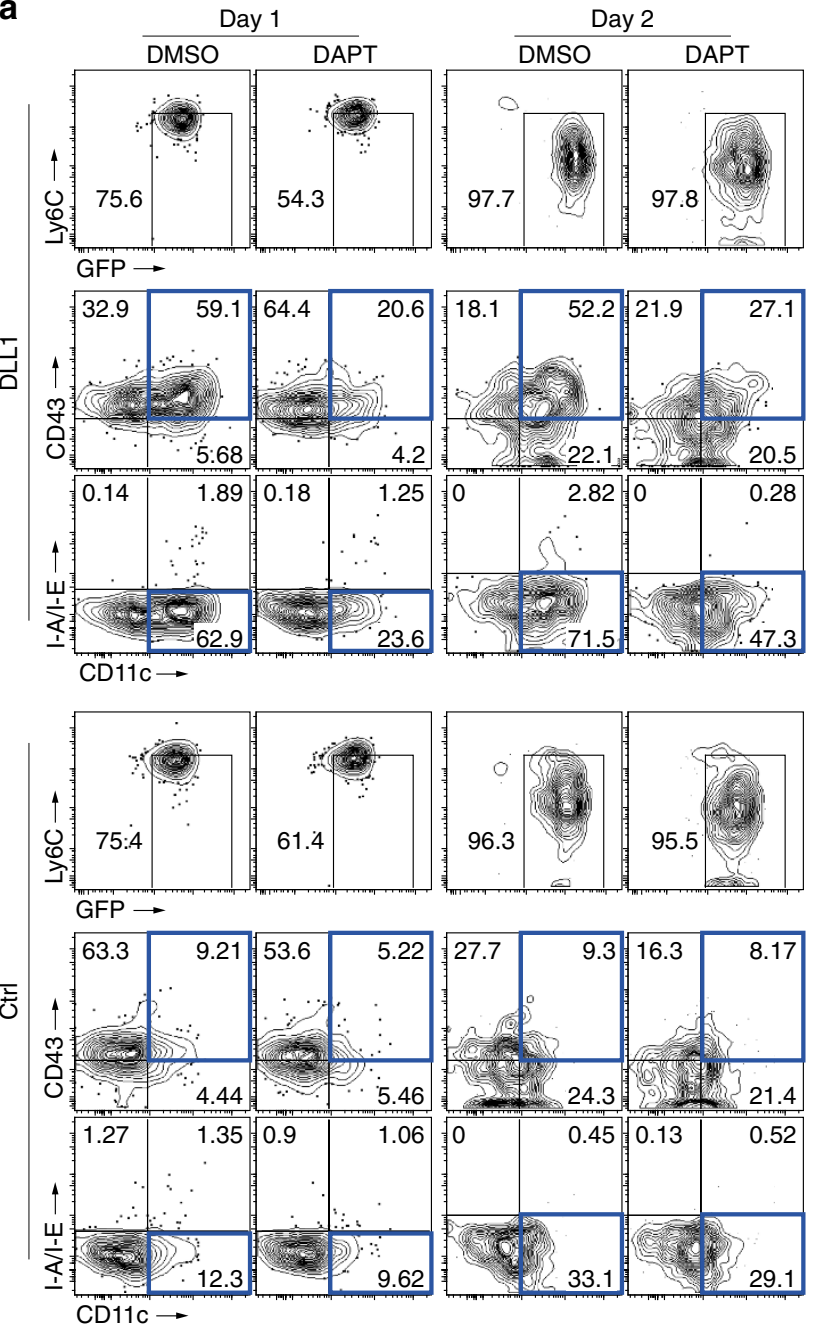

b

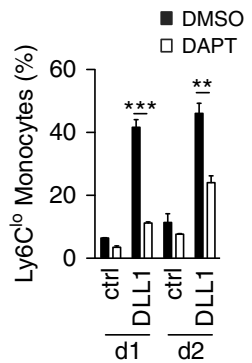

C

CD11b ${ }^{+} \mathrm{GFP}^{+} \mathrm{Ly} 6 \mathrm{C}^{\mathrm{lo} /-}$ gate:

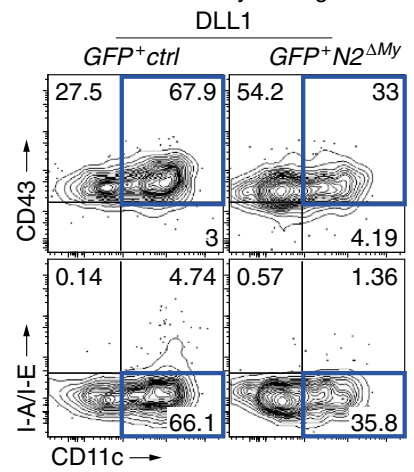

d

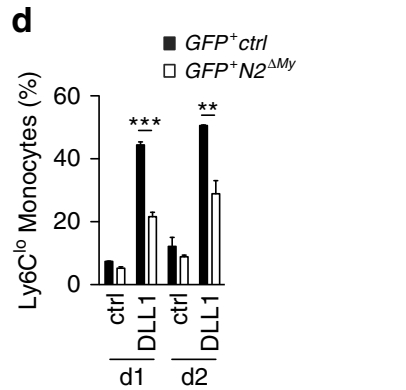

Figure 6 | Notch2 signalling is required for monocyte conversion in vitro. (a,b) Inhibition of Notch signalling in sorted Ly6Chi monocytes using a $\gamma$-secretase inhibitor (DAPT) impairs conversion in vitro. Representative flow cytometry plot from live $\mathrm{CD}_{11 \mathrm{~b}}{ }^{+} \mathrm{GFP}^{+}$cells (a) and relative frequency (b) of Ly6Clo monocyte-like cells (CD11b + GFP + Ly6C $\mathrm{Cl}^{\mathrm{lo} /-} \mathrm{CD} 11 \mathrm{C}^{\mathrm{lo}} \mathrm{CD} 43^{+} \mathrm{MHC}-\mathrm{II}^{\mathrm{lo} /-}$ ) are shown. (c,d) Ly6Chi monocytes from GFP+ Notch2 ${ }^{4 M y}$ mice show impaired conversion. Representative flow cytometry plot (c) and relative frequency (d) of Ly6 $6 \mathrm{C}^{\text {lo }}$ monocyte-like cells are shown (similar to Fig. 6a,b). (b,d) $n=3,{ }^{\star} P<0.05,{ }^{* \star} P<0.01,{ }^{\star \star \star} P<0.001$; one-way ANOVA with Bonferroni's Multiple comparison test. Error bars represent s.e.m.

demonstrated Cre activity in splenic MZ EC and BM EC (Fig. 8a, Supplementary Fig. 9c). Employing a conditional allele of Dll1 (ref. 23) we next generated endothelial-specific and inducible Dll1 mutant mice $\left(D l l 1^{i \Delta E C}\right)$ and confirmed Cre-dependent recombination and deletion of the conditional Dll1 allele after a pulse of tamoxifen (Supplementary Fig. 9d,e). In controlled experiments (Supplementary Fig. 7f,g), endothelial deletion of Dll1 resulted in the selective reduction of Ly6C $\mathrm{C}^{\text {lo }}$ monocytes, while Ly6 $\mathrm{C}^{\text {hi }}$ monocytes and monocyte progenitors where unchanged compared with control (Fig. 8b). These data demonstrate that endothelial Dll1 regulates Ly6C $\mathrm{C}^{\mathrm{lo}}$ monocytes in vivo.

Although our data clearly indicated the importance of endothelial Dll1 for monocyte conversion, given the selective expression of Dll1 in two distinct endothelial domains, arteries and the haematopoietic compartment (BM and spleen), the identity of the endothelial domain mediating monocyte conversion remained unclear and could not be addressed with our pan-endothelial deletion strategy. We, therefore, generated mice with inducible, but arterial EC-specific deletion of Dll1, by crossing the floxed allele of Dll1 to $B m x(P A C)-C r e E R T 2$ mice $\left(D l l 1^{i \triangle a E C}\right)^{39}$. We confirmed the arterial EC-specific Cre activity in a Cre-dependent lacZ-reporter strain (lacZiaEC), which demonstrated specific EC staining in aorta, peripheral arteries and central arteries of the splenic follicles, while MZ EC were not targeted, thus providing a tool to address the contribution of arterial EC to monocyte conversion (Supplementary Fig. 10a). We induced Dll1 deletion in arterial EC, which resulted in Cre-dependent recombination of the conditional Dll1 allele (Supplementary Fig. 10b). In contrast to pan-endothelial deletion of Dll1, arterial EC-specific deletion of Dll1 did not affect relative or absolute numbers of Ly6C ${ }^{\text {lo }}$ monocytes (Fig. 8c), suggesting that Dll1 expressed in endothelial niches in the MZ or $\mathrm{BM}$, and not in arterial endothelium, regulates monocyte conversion.

To further investigate the specificity of Dll1 effects on Ly6C $\mathrm{C}^{\text {lo }}$ monocytes we mated the pan-endothelial and inducible Cre-transgenic strain to conditional alleles of the related Notch ligand Dll4 (refs 38,40). In contrast to deletion of Dll1, deletion of Dll4 did not lead to alterations in Ly6 $\mathrm{C}^{\text {lo }}$ monocytes, or any other myeloid subset (Fig. 8d), which demonstrates specific effects of Dll1 in the regulation of Ly6 $\mathrm{C}^{\text {lo }}$ monocytes.

To provide proof-of-principle that EC populations from the haematopoietic compartment regulate monocyte conversion we established an in vitro co-culture system. CD144 ${ }^{+}$ECs 
a
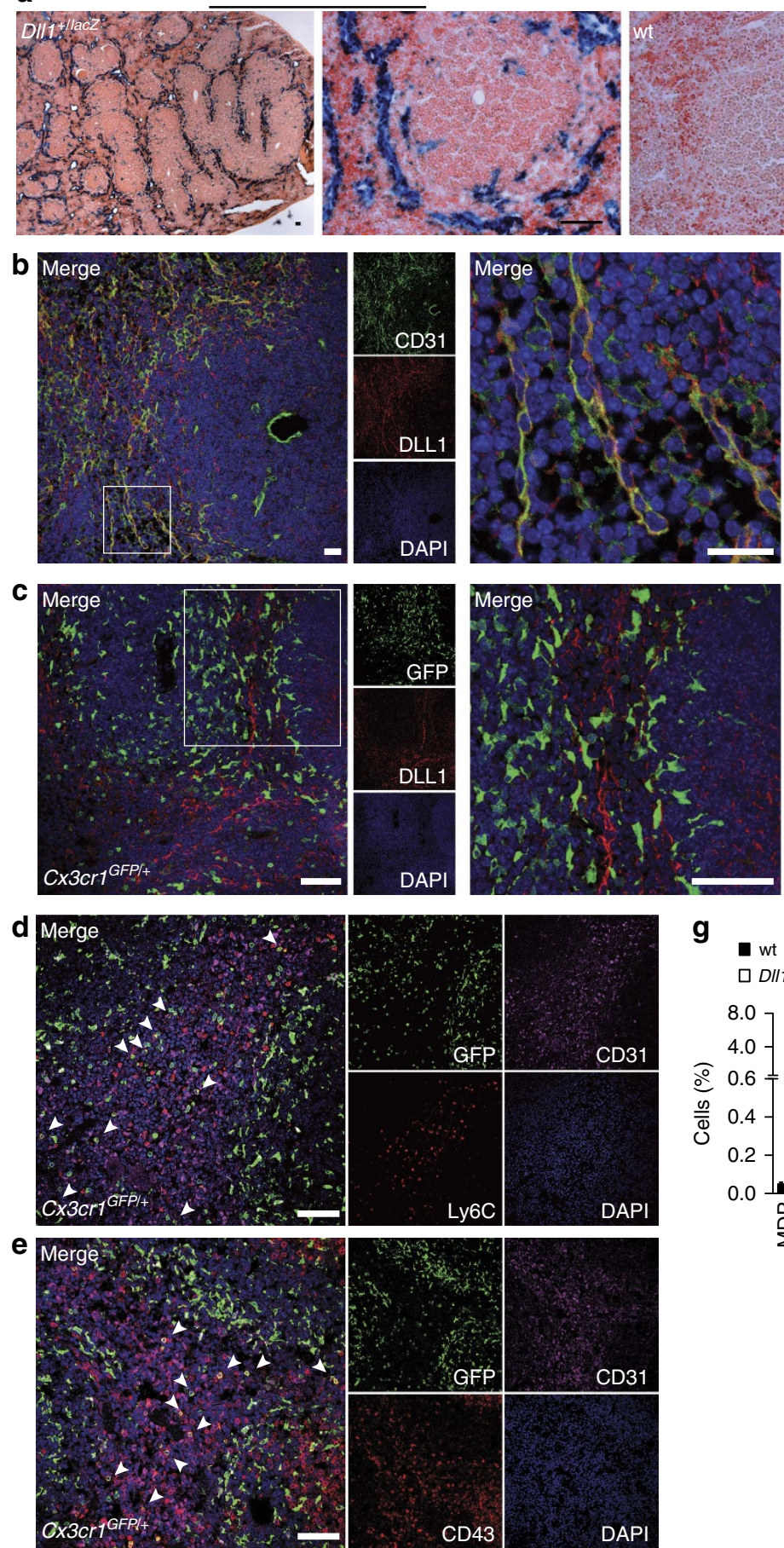
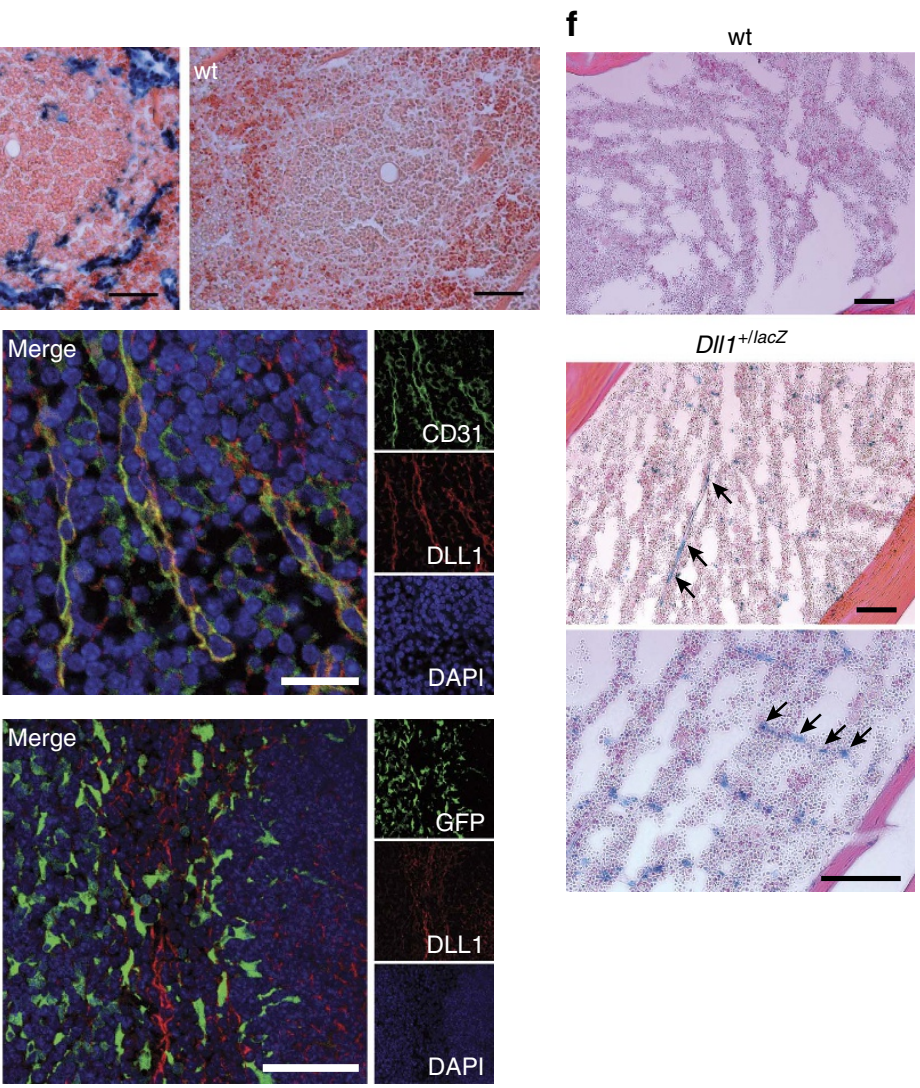

g
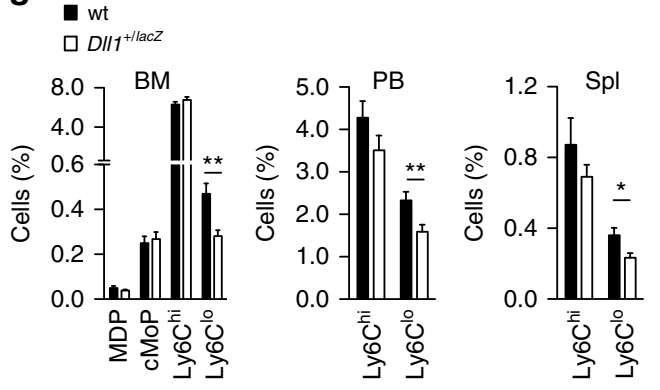

Figure 7 | DII1 deficiency impairs Ly6Clo monocyte development in vivo. (a) MZ-specific $\beta$-galactosidase activity in splenic follicle of DII1+/lacZ mice (left), but not in wt control (right). Scale bars, $50 \mu \mathrm{m}$. (b) Immunostaining and confocal microscopy of splenic follicle MZ demonstrating DLL1 expression in $\mathrm{CD} 1^{+} \mathrm{EC}$. Scale bar, $20 \mu \mathrm{m}$. (c) Immunostaining and confocal microscopy showing GFP ${ }^{+}$cells in association with DLL1 ${ }^{+}$structures in splenic follicle MZ.

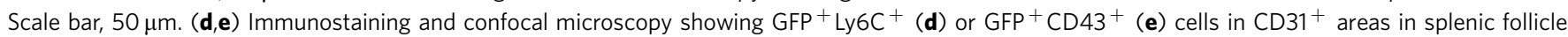
MZ. Large GFP ${ }^{+}$cells bordering MZ are Ly6C or CD43 negative. Scale bar, $50 \mu \mathrm{m}$. (f) DIl1 expression in BM of femur diaphysis indicated by specific

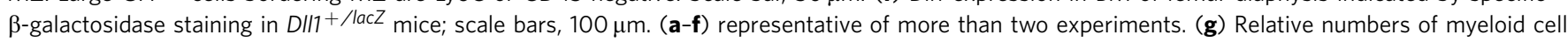

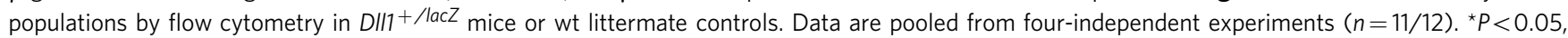
${ }^{\star \star} P<0.01,{ }^{\star \star \star} P<0.001$; Student's $t$-test. Error bars represent s.e.m.

were sorted from splenic tissue digests and cultured with Ly6 $\mathrm{Chi}^{\text {hi }}$ monocytes from $C x 3 \mathrm{cr} 1^{G F P /+}$ reporter mice. We also confirmed Dll1 expression in this EC population (Fig. 8e). Compared with monocytes cultured alone, conversion of Ly6 $\mathrm{C}^{\text {hi }}$ monocytes into Ly6C ${ }^{\text {lo }}$ monocyte-like cells was significantly increased on splenic EC, an effect that persisted over time (Fig. 8f,g). Altogether, these results suggest that distinct endothelial niches in the haematopoietic compartments regulate conversion of Ly6 $\mathrm{C}^{\text {hi }}$ monocytes into Ly6C $\mathrm{C}^{\mathrm{lo}}$ monocytes via the Dll1-Notch2 axis. 
a

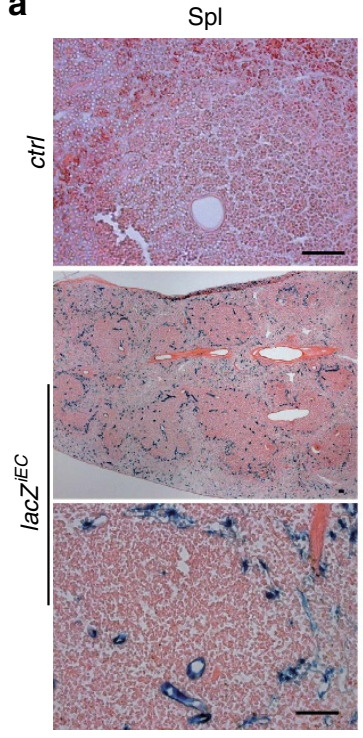

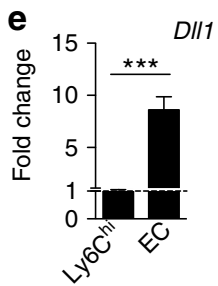

BM
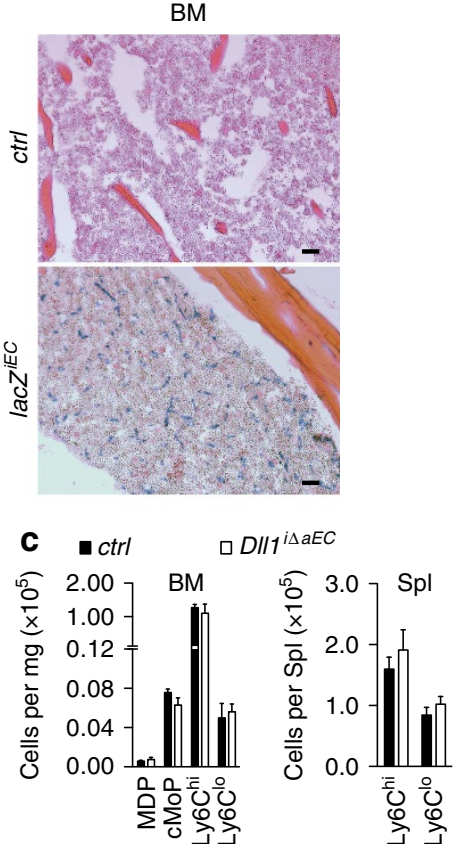
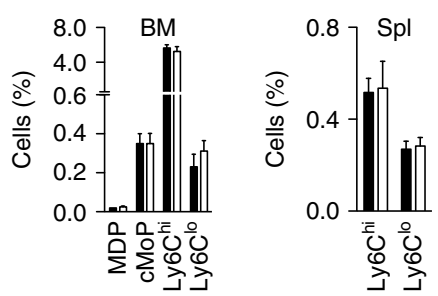

f
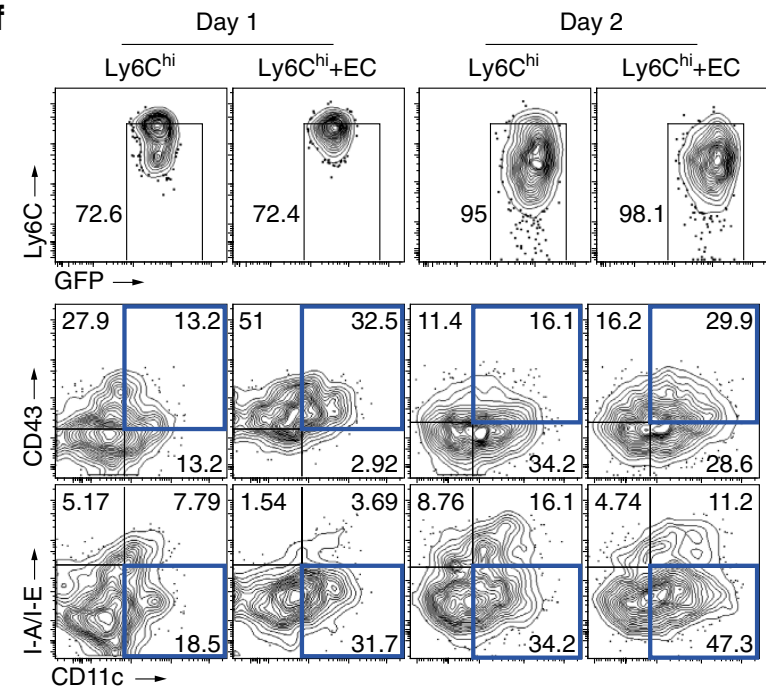

b
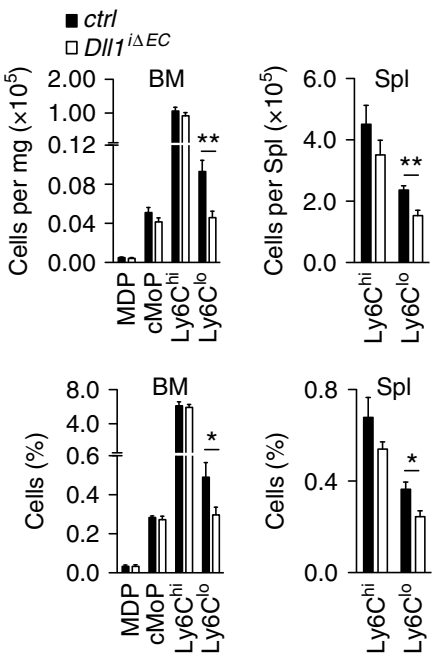

d
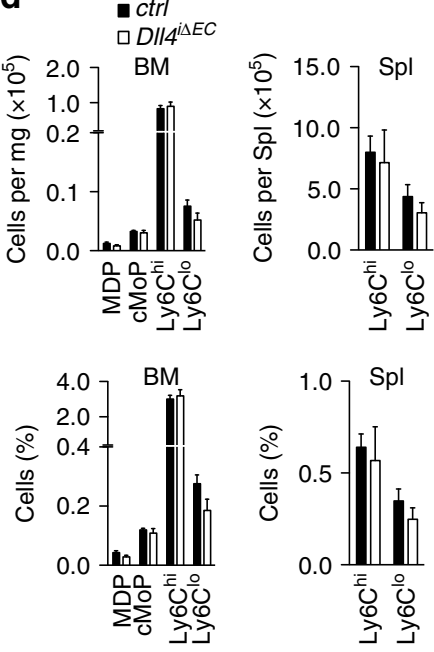

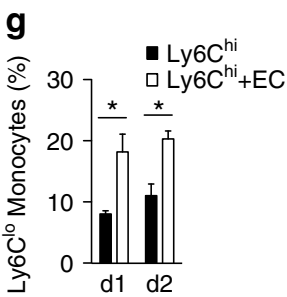

Figure 8 | Endothelial deletion of DII1 impairs Ly6 $\mathbf{C}^{\mathbf{l o}}$ monocyte development in vivo. (a) Inducible and endothelial-specific Cre-reporter mice (lacZiEC) showing specific $\beta$-galactosidase staining in splenic MZ (left) and BM (right) of the femur diaphysis after tamoxifen treatment; scale bars, $50 \mu \mathrm{m}$.

(b) Absolute and relative numbers of myeloid cell populations by flow cytometry after pan-endothelial deletion of DII1 compared with littermate controls. Data are pooled from four experiments $(n=8)$. (c) Absolute and relative numbers of myeloid cell populations by flow cytometry after arterial-specific, endothelial deletion of DII1 compared with littermate controls. Data are pooled from two experiments $(n=5 / 6)$. (d) Absolute and relative numbers of myeloid cell populations by flow cytometry after endothelial-specific deletion of DII4 compared with littermate controls. Data are pooled from two experiments $(n=5)$. (e) Quantitative reverse transcription-PCR analysis of DI11 expression in sorted splenic monocytes $\left(\right.$ CD11b $\left.{ }^{+} \mathrm{GFP}^{+} \mathrm{Ly}_{6 \mathrm{C}^{\text {hi }}} \mathrm{CD} 144^{-}\right)$ and ECs $\left(\mathrm{CD} 11 b^{-} \mathrm{GFP}^{-} \mathrm{CD}_{144^{+}}\right)$. Data are pooled from three-independent experiments $(n=6)$. (f,g) Co-culture of splenic CD144 ${ }^{+}$ECs with Ly6Chi monocytes. Representative flow cytometry plot from GFP ${ }^{+} \mathrm{CD}_{11 \mathrm{~b}}{ }^{+}$gate $(\mathbf{f})$ and frequency of Ly6 $\mathrm{C}^{\mathrm{lo}}$ monocyte-like cells $(\mathbf{g})$ are shown $(n=3)$. ${ }^{\star} P<0.05,{ }^{\star \star} P<0.01,{ }^{\star \star \star} P<0.001$; Student's $t$-test $(\mathbf{b}-\mathbf{e})$ or one-way ANOVA with Bonferroni's multiple comparison test (g). (b-e,g) Error bars represent s.e.m. 


\section{Discussion}

We here define the molecular and cellular context that regulates monocyte conversion. Taken together, our results show that Notch2 regulates conversion of Ly6 $\mathrm{C}^{\text {hi }}$ monocytes into Ly6Clo monocytes, thereby regulating a specific developmental step in monocyte cell fate under steady-state conditions. This process is controlled specifically by Notch ligand Dll1 expressed by a population of EC that constitute distinct vascular niches in the BM and spleen. Thus, blood vessels regulate monocyte conversion, as a form of developmental cell fate regulation.

The origin and regulation of Ly6 $\mathrm{C}^{\text {lo }}$ monocytes is still poorly understood. Recent findings have suggested that Ly6Clo monocytes develop from Ly6C ${ }^{\text {hi }}$ monocytes. Adoptive transfer of cMoP leads to the sequential appearance of Ly6Chi monocytes followed by Ly6C lo monocytes ${ }^{3}$. Furthermore, Jung and colleagues have provided direct evidence that Ly6C ${ }^{\text {lo }}$ monocytes derive from Ly6C $\mathrm{C}^{\text {hi }}$ monocytes by adoptive transfer experiments $^{7,14}$. Our experiments demonstrating monocyte conversion with isolated Ly6Chi monocytes in vivo, and recapitulation of it in vitro, clearly support the notion of monocyte conversion as a mechanism regulating development of Ly6C $\mathrm{C}^{\text {lo }}$ monocytes. However, while our data provide evidence for and insights into the mechanism of monocyte conversion, our findings do not exclude the existence of additional mechanisms to generate $\mathrm{Ly}^{\mathrm{l}} \mathrm{C}^{\text {lo }}$ monocytes, for example from progenitor cells, as has been recently suggested ${ }^{41}$.

So far, specific molecular or cellular regulators of monocyte conversion have remained elusive, and it has been speculated that monocyte conversion occurs spontaneously in the circulation ${ }^{2,14}$. Our data demonstrating a requirement for the Dll1-Notch2 signalling axis for the conversion of Ly6 $\mathrm{C}^{\text {hi }}$ monocytes into Ly6C $C^{\text {lo }}$ monocytes not only provides clear evidence for the regulated nature of monocyte conversion, but also shows that this process is under control of blood vessels through Notch ligand Dll1. The fact that conditional deletion of Dll4 has no impact on Ly6C $\mathrm{C}^{\text {lo }}$ monocytes further emphasizes the specific nature of Dll1 actions. Although the precise location of monocyte conversion remains unknown, our data clearly demonstrate the existence, and functional importance, of distinct Dll1-expressing vascular niches in BM and spleen. This suggests that monocyte conversion happens, or is at least initiated, in these vascular niches under steady-state conditions. This is further supported by the fact that co-cultured EC from these niches promote monocyte conversion, while our in vivo data from mice with arterial-specific Dll1 deletion exclude the participation of arterial ECs in this process. However, given the dynamic nature of endothelial responses, it is conceivable that in certain situations, such as inflammation, the location, composition and extent of vascular niches might change, which, in turn, might influence monocyte conversion rates.

Recently, the instructive function of the vascular niche for self-renewal and regenerative capacity of HSCs has been defined, which, in part, is mediated by endothelial-specific expression of Notch ligand Jag1 (refs 20,21). Our finding that monocyte conversion is regulated by ECs extends the spectrum of niche regulation towards more committed steps of myeloid cell development and further supports the importance of the vascular niche in regulating cell fate. On the other hand, the finding that monocyte conversion is specifically regulated by vascular Dll1 underlines the ligand-specific nature of Notch signalling events in different locations ${ }^{16}$.

Our data also suggest that Notch2 expressed in monocytes is directly involved in regulation of Ly6 $\mathrm{C}^{\mathrm{lo}}$ monocyte cell fate in response to Dll1. Several lines of evidence support this conclusion. First, Notch2 was required for monocyte conversion in experiments using isolated Ly6C hi monocytes in vivo and in vitro. Second, when Notch 2 was targeted on Ly6C hi monocytes in vivo we found that the extent of Notch2 loss-of-function in Ly6 $\mathrm{C}^{\text {hi }}$ monocytes is related to the loss of Ly6C $\mathrm{C}^{\mathrm{lo}}$ monocytes, while Ly6C ${ }^{\text {hi }}$ monocyte numbers are not affected by Notch2 deletion. On the other hand, targeting of Notch2 in Ly6C $\mathrm{C}^{\mathrm{lo}}$ monocytes also resulted in selective deletion of $\mathrm{Ly}_{6 \mathrm{C}} \mathrm{l}^{\mathrm{o}}$ monocytes. Together, this demonstrates a requirement for monocyte Notch2 in the generation, as well as maintenance of Ly6C ${ }^{\text {lo }}$ monocytes. Finally, Notch2 loss-of-function lead to the appearance of an atypical monocyte population negative for CD11c and CD43 but expressing high levels of MHC-II and CCR2. These findings suggest a model in which Notch2 regulates $\mathrm{Ly}_{6 \mathrm{C}} \mathrm{C}^{\mathrm{lo}}$ monocyte cell fate: active Notch2 signalling mediates conversion into Ly6 $\mathrm{C}^{\text {lo }}$ monocytes, while defective Notch2 signalling leads to MHC-II ${ }^{\text {hi }}$ atypical monocytes. While the role and relevance of this atypical monocyte population observed in conditional mutants is currently unclear, it is important to note that, using different gating strategies and experimental approaches, subpopulations of MHC-II-expressing monocytes have recently been described, which acquire antigen for carriage to lymph nodes ${ }^{36}$.

Currently, the molecular effectors of Notch2 in monocytes are unknown. Interestingly, the transcription factor $\mathrm{Nr} 4 \mathrm{a} 1$, an orphan nuclear receptor, regulates the survival of Ly6 $\mathrm{C}^{\text {lo }}$ monocytes ${ }^{13}$. Although a role of Nr4al in monocyte conversion has not been investigated, mice with general or BM-restricted inactivation of $\mathrm{Nr} 4 \mathrm{a} 1$ showed reduced Ly $6 \mathrm{C}^{\text {lo }}$ monocytes numbers and increased rates of apoptosis. Although we did not observe a cell death phenotype in Notch 2 mutant mice, we found DLL1-dependent regulation of Nr4al in vitro. Clearly, the molecular regulation of monocyte conversion requires further study.

Our study also describes the first steps towards an approach to recapitulate monocyte cell fate ex vivo. This might provide a setting to study and understand the molecular events driving monocyte conversion under defined conditions.

\section{Methods}

Mice. Mouse strains used in the study are listed in Supplementary Table 2. Cx3cr $1^{\text {GFP } /+}$ mice $^{28}$ (kindly provided by Steffen Jung), Lys $M^{\text {Cre }}$ mice $^{32}$, Dll1 $1^{+/ l a c Z}$ mice $^{42}$ (kindly provided by Achim Gossler), Notch $1^{\text {lox/lox }}$ and Notch $2^{\text {lox } / l o x}$ mice ${ }^{17}$, Cdh5(PAC)-CreERT2 mice ${ }^{43}, B m x(P A C)-C r e E R T 2$ mice $^{44}, D l l 1^{\text {lox/lox }}$ mice $^{23}$,

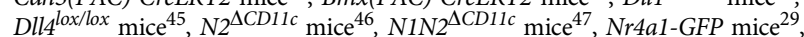
LysM-eGFP mice ${ }^{33}$, Lys $M^{\text {Cre }}$ Rosa $^{\text {YFP }}$ mice ${ }^{48}$ have been described. Gt(ROSA)26Sor mice carrying Cre-inducible lac $Z$ alleles were obtained from The Jackson Laboratories, B6.SJL-Ptprc ${ }^{a} P e p c^{b} /$ BoyJ $\left(\mathrm{CD} 45.1^{+}\right)$mice from Charles River. Mice were housed under specific pathogen free conditions in the animal facility of Hannover Medical School unless otherwise indicated. Nr4a1-GFP, LysM-eGFP and $L y s M^{C r e} R_{0 s a}{ }^{Y F P}$ mice were housed in IPEC, Munich, Germany; $N 2^{\triangle C D 11 c}$ mice in WUSTL, St Louis, MO, USA; N1N2 $2^{\Delta C D 11 c}$ mice in Tokushima University, Tokushima, Japan and Cdh5(PAC)-CreERT2 Dll4 $4^{\text {lox/lox }}$ mice in MPI Munster, Germany. All experiments were performed with 8-12-weeks-old mice and age and sex matched littermate controls with approval of the local animal welfare boards (LAVES Lower Saxony, Animal Studies Committee at Washington University in St Louis, Animal Research Committee of Tokushima University, North Rhine Westphalia Animal Ethics Committee and Local Animal Committee of District Government of Upper Bavaria).

Tissue and cell preparation. For single cell suspension mice were killed and spleen, BM and blood were collected. Erythrocytes were removed by red blood cell lysis buffer (Biolegend) or by density centrifugation using Histopaque 1083 (SigmaAldrich). After extensive washing cells were resuspended in PBS containing 10\% FCS and 2 mM EDTA kept on ice, stained and used for flow cytometry or for sorting.

Flow cytometry and cell sorting. Non-specific binding of antibodies by Fc-receptors was blocked with anti-mouse CD16/CD32 (TruStain fcX from Biolegend) in single cell suspensions prepared from spleen, PB or BM. After subsequent washing step cells were labelled with primary and secondary antibodies or streptavidin-fluorochrome conjugates (Supplementary Table 3) and used for flow cytometry analysis (LSR-II, BD Biosciences) or sorting (FACSAria; BD Biosciences or MoFlo XDP; Beckman Coulter). Data were analysed by FlowJo software (Treestar). Initially cells were identified based on forward scatter (FSC) and side scatter (SSC) characteristics. After exclusion of doublets (on the basis of 
SSC-W, SSC-A), relative frequency of each subpopulation from live cell gate or absolute number of each subset (calculated from live cell gate and normalized on $\mathrm{mg} \mathrm{BM}, \mu \mathrm{PB}$ or spleen) were determined and are shown in the graphs as mean \pm s.e.m., unless otherwise stated.

In vitro conversion studies. Ninety-six-well flat bottom plates were coated at room temperature for $3 \mathrm{~h}$ with IgG-Fc, JAG1-Fc or DLL1-Fc ligands (all from R\&D) reconstituted in PBS. Sorted BM Ly6 $\mathrm{C}^{\text {hi }}$ monocytes were cultured in coated plates in the presence of M-CSF $\left(10 \mathrm{ng} \mathrm{ml}^{-1}\right)$, thrombopoietin (TPO, $20 \mathrm{ng} \mathrm{ml}^{-1}$ ), stem cell factor (SCF, $10 \mathrm{ng} \mathrm{ml}^{-1}$ ), insulin-like growth factor (IGF)-II $\left(20 \mathrm{ng} \mathrm{ml}^{-1}\right.$ ), fibroblast growth factor (FGF)-I (10 $\mathrm{ng} \mathrm{ml}^{-1}$ ) (all from Peprotech) and Heparin $\left(25 \mathrm{U} \mathrm{ml}^{-1}\right)$ at $37^{\circ} \mathrm{C}$ for 24 or $48 \mathrm{~h}$. In experiments where the effect of Notch inhibition on conversion process was assessed, $6 \mu \mathrm{M}$ of $\gamma$-secretase inhibitor, DAPT or dimethylsulphoxide (DMSO) was applied to the cells prior to and $24 \mathrm{~h}$ after culture. In separate experiments BM Ly6 $\mathrm{C}^{\mathrm{hi}}$ monocytes were co-cultured with sorted splenic $\mathrm{CD}_{144^{+}} \mathrm{GFP}^{-} \mathrm{CD}_{11 \mathrm{~b}}{ }^{-} \mathrm{EC}$. One or 2 days after culture, cells were harvested, stained and subjected to flow cytometry. Frequency of Ly6C $\mathrm{C}^{\mathrm{lo}}$ monocyte-like cells $\left(\mathrm{CD}_{11} \mathrm{~b}^{+} \mathrm{GFP}^{+} \mathrm{Ly} \mathrm{C}^{\mathrm{lo} /}-\mathrm{CD} 11 \mathrm{c}^{\mathrm{lo}} \mathrm{MHC}-\mathrm{II}^{\mathrm{lo} /}-\mathrm{CD} 43^{+}\right)$in total live $\mathrm{CD} 11 \mathrm{~b}^{+}$ $\mathrm{GFP}^{+}$cells served as an indicator of conversion efficiency and is shown in the graphs.

Adoptive cell transfer experiments. $\mathrm{CD}_{11} \mathrm{~b}^{+} \mathrm{Ly}_{6} \mathrm{C}^{\mathrm{hi}} \mathrm{GFP}{ }^{+}$monocytes were sorted from $\mathrm{BM}$ and injected into $\mathrm{CD} 45.1^{+}$recipients intravenously (i.v.). Four days after transfer spleen, PB and BM were collected and single cell suspension was prepared. After blocking of Fc receptors using anti-mouse CD16/CD32 (TruStain fcX from Biolegend) cells were labelled with biotin-conjugated anti-CD45.1 antibody, anti-biotin magnetic beads and enriched on LD columns (Miltenyi Biotec) according to the manufacturer's instructions. CD45.1 negative fraction was collected, stained and analysed by flow cytometry. Ly6 $\mathrm{C}^{\mathrm{lo}}$ monocytes $\left(\mathrm{CD} 11 \mathrm{~b}{ }^{+}\right.$ $\mathrm{GFP}^{+} \mathrm{Ly} 6 \mathrm{C}^{\mathrm{lo} /}-\mathrm{F} 4 / 80^{\mathrm{lo}} \mathrm{CD} 11 \mathrm{c}^{\mathrm{lo}} \mathrm{MHC}-\mathrm{II}^{\mathrm{lo} /}-$ cells) were quantified in spleen, BM and $\mathrm{PB}$ as relative frequency of total donor derived $\mathrm{CD} 45.2^{+} \mathrm{CD} 11 \mathrm{~b}^{+} \mathrm{GFP}^{+}$cells.

Human monocyte isolation. Human monocytes were isolated from the blood of healthy individuals as approved by the ethical committee of Hannover Medical School. Written consent was obtained before blood collection. Cells were purified using $\mathrm{CD}_{16}{ }^{+}$monocyte isolation kit (Miltenyi Biotec) according to the manufacturer's instruction. $\mathrm{CD}_{14}{ }^{+}$monocytes were retrieved in subsequent purification step from CD16 ${ }^{\text {neg }}$ fraction using CD14 microbeads.

\section{Immunohistochemistry and immunofluorescence. Immunohistochemistry,} $\beta$-galactosidase and immunofluorescence staining in mice were performed with modifications from previous descriptions ${ }^{37,49}$. Mice were euthanized; tibiae, spleen, heart, aorta and muscles were isolated and fixed in $4 \%$ paraformaldehyde (PFA). Bones were decalcified in $0.5 \mathrm{M}$ EDTA solution at $4{ }^{\circ} \mathrm{C}$ for $48 \mathrm{~h}$, cryopreserved in sucrose and embedded in Tissue-tek O.C.T. compound (Sakura, Germany). All the other organs were cryoprotected in sucrose and embedded in Tissue-tek O.C.T. compound without decalcification procedure. $\beta$-galactosidase staining was performed at $37^{\circ} \mathrm{C}$ on PFA fixed tissues. Slides were counterstained with eosin, mounted in mounting medium and analysed with Olympus IX71 microscope. For immunofluorescence and confocal laser scanning microscopy tissue sections were stained using anti-DLL1 (Biolegend), anti-CD31, anti-CD43 (both from BD Biosciences, Germany), anti-Ly6C (Biolegend) and appropriate fluorescenceconjugated secondary antibodies (Supplementary Table 3). 4,6-Diamidino-2phenylindole (Invitrogen, Germany) was used for counterstaining of nuclei and slides were mounted in DAKO fluorescence mounting medium (Dako, Denmark). Images were acquired using Leica TCS SP2 AOBS (Leica Microsystems, Germany) confocal microscope or Zeiss Observer Z1 fluorescence microscope (Zeiss, Germany), respectively.

Intravital microscopy. To visualize monocytes in the microcirculation the cremaster muscle of male $G F P^{+} c t r l$ and $G F P^{+} N 2^{4 M y}$ mice was exposed and transient and adhesive interactions were recorded. To this end an Olympus BX51 microscope equipped with a Hamamatsu 9100-02 EMCCD camera (Hamamatsu Photonics) and a $\times 40$ water-dipping objective was employed. In each cremaster 10 fields of view were recorded for $30 \mathrm{~s}$ and the number of adherent cells and the rolling flux (rolling monocytes passing a perpendicular line placed across the observed vessel) from each field were quantified. Subsequent to recordings at baseline, mice were injected via a jugular vein catheter with a single dose of TNF- $\alpha$ $(250 \mathrm{ng})$ and recordings were repeated after $60 \mathrm{~min}$.

Quantitative real-time PCR analysis. Splenic ECs, as well as splenic or BM monocytes were isolated by cell sorting and total RNA was purified using Nucleospin RNA II kit (Macherey Nagel). After purity and quality check, RNA was transcribed into complementary DNA employing complementary DNA synthesis kit (Invitrogen) according to the manufacturer's instructions. Quantitative real-time PCR was performed using specific primers (Supplementary Table 4) and FastStart Essential DNA Green Master on a LightCycler 96 system from
Roche according to the manufacturer's instructions, for normalization. Expression of each specific gene was normalized to expression of Rps 9 and calculated by the comparative CT $\left(2^{-\Delta \Delta \mathrm{CT}}\right)$ method $^{50}$.

In vivo targeting of EC and PCR analysis. Five to 6-weeks-old mice expressing EC-specific inducible Cre recombinase and Cre-negative littermate controls were treated with $500 \mu \mathrm{g}$ tamoxifen for 5 consecutive days intraperitoneally (i.p.). Mice were killed after 4 weeks. Efficiency of Cre-dependent gene deletion was monitored by PCR and agarose gel-electrophoresis as described ${ }^{23}$.

Statistical analysis. Results are expressed as mean \pm s.e.m.. $N$ numbers are biological replicates of experiments performed at least three times unless otherwise indicated. Significance of differences was calculated using unpaired, two-tailed Student's $t$-test with confidence interval of $95 \%$. For comparison of multiple experimental groups one-way analysis of variance (ANOVA) was used and Bonferroni's multiple-comparison test was performed when the overall $P$ value was $<0.05$. Data from intravital microscopy were analysed using two-way ANOVA and Bonferroni's post-test. $P$ values of less than 0.05 were considered to be significant.

Data availability. The authors declare that all the relevant data are available upon request.

\section{References}

1. Geissmann, F., Jung, S. \& Littman, D. R. Blood monocytes consist of two principal subsets with distinct migratory properties. Immunity 19, 71-82 (2003).

2. Ginhoux, F. \& Jung, S. Monocytes and macrophages: developmental pathways and tissue homeostasis. Nat. Rev. Immunol. 14, 392-404 (2014).

3. Hettinger, J. et al. Origin of monocytes and macrophages in a committed progenitor. Nat. Immunol. 14, 821-830 (2013).

4. Fogg, D. K. et al. A clonogenic bone marrow progenitor specific for macrophages and dendritic cells. Science 311, 83-87 (2006).

5. Nahrendorf, M. et al. The healing myocardium sequentially mobilizes two monocyte subsets with divergent and complementary functions. J. Exp. Med. 204, 3037-3047 (2007).

6. Auffray, C. et al. CX3CR1 + CD115 + CD135 + common macrophage/DC precursors and the role of CX3CR1 in their response to inflammation. J. Exp. Med. 206, 595-606 (2009).

7. Varol, C. et al. Monocytes give rise to mucosal, but not splenic, conventional dendritic cells. J. Exp. Med. 204, 171-180 (2007).

8. Auffray, C. et al. Monitoring of blood vessels and tissues by a population of monocytes with patrolling behavior. Science 317, 666-670 (2007).

9. Cros, J. et al. Human CD14dim monocytes patrol and sense nucleic acids and viruses via TLR7 and TLR8 receptors. Immunity 33, 375-386 (2010).

10. Carlin, L. M. et al. Nr4a1-dependent Ly6C(low) monocytes monitor endothelial cells and orchestrate their disposal. Cell 153, 362-375 (2013).

11. Biburger, M. et al. Monocyte subsets responsible for immunoglobulin G-dependent effector functions in vivo. Immunity 35, 932-944 (2011).

12. Santiago-Raber, M.-L. et al. Fcgamma receptor-dependent expansion of a hyperactive monocyte subset in lupus-prone mice. Arthritis Rheum. 60, 2408-2417 (2009).

13. Hanna, R. N. et al. The transcription factor NR4A1 (Nur77) controls bone marrow differentiation and the survival of Ly6C- monocytes. Nat. Immunol. 12, 778-785 (2011).

14. Yona, S. et al. Fate mapping reveals origins and dynamics of monocytes and tissue macrophages under homeostasis. Immunity 38, 79-91 (2013).

15. Radtke, F., Macdonald, H. R. \& Tacchini-Cottier, F. Regulation of innate and adaptive immunity by Notch. Nat. Rev. Immunol. 13, 427-437 (2013).

16. Benedito, R. et al. The Notch ligands Dll4 and jagged1 have opposing effects on angiogenesis. Cell 137, 1124-1135 (2009).

17. Besseyrias, V. et al. Hierarchy of Notch-Delta interactions promoting $T$ cell lineage commitment and maturation. J. Exp. Med. 204, 331-343 (2007).

18. Morrison, S. J. \& Scadden, D. T. The bone marrow niche for haematopoietic stem cells. Nature 505, 327-334 (2014).

19. Butler, J. M., Kobayashi, H. \& Rafii, S. Instructive role of the vascular niche in promoting tumour growth and tissue repair by angiocrine factors. Nat. Rev Cancer 10, 138-146 (2010).

20. Butler, J. M. et al. Endothelial cells are essential for the self-renewal and repopulation of Notch-dependent hematopoietic stem cells. Cell Stem Cell 6, 251-264 (2010).

21. Poulos, M. G. et al. Endothelial jagged-1 is necessary for homeostatic and regenerative hematopoiesis. Cell Rep. 4, 1022-1034 (2013).

22. Cao, Z. et al. Angiocrine factors deployed by tumor vascular niche induce B cell lymphoma invasiveness and chemoresistance. Cancer Cell 25, 350-365 (2014).

23. Hozumi, K. et al. Delta-like 1 is necessary for the generation of marginal zone B cells but not T cells in vivo. Nat. Immunol. 5, 638-644 (2004). 
24. Tan, J. B. et al. Lunatic and manic fringe cooperatively enhance marginal zone B cell precursor competition for delta-like 1 in splenic endothelial niches. Immunity 30, 254-263 (2009).

25. Fasnacht, N. et al. Specific fibroblastic niches in secondary lymphoid organs orchestrate distinct Notch-regulated immune responses. J. Exp. Med. 211, 2265-2279 (2014).

26. Ohishi, K., Varnum-Finney, B., Serda, R. E., Anasetti, C. \& Bernstein, I. D. The Notch ligand, Delta-1, inhibits the differentiation of monocytes into macrophages but permits their differentiation into dendritic cells. Blood 98, 1402-1407 (2001).

27. Ingersoll, M. A. et al. Comparison of gene expression profiles between human and mouse monocyte subsets. Blood 115, e10-e19 (2010).

28. Jung, S. et al. Analysis of fractalkine receptor CX(3)CR1 function by targeted deletion and green fluorescent protein reporter gene insertion. Mol. Cell. Biol. 20, 4106-4114 (2000).

29. Moran, A. E. et al. T cell receptor signal strength in Treg and iNKT cell development demonstrated by a novel fluorescent reporter mouse. J. Exp. Med. 208, 1279-1289 (2011).

30. Maillard, I., Fang, T. \& Pear, W. S. Regulation of lymphoid development, differentiation, and function by the Notch pathway. Annu. Rev. Immunol. 23, 945-974 (2005).

31. Radtke, F. et al. Deficient T cell fate specification in mice with an induced inactivation of Notch1. Immunity 10, 547-558 (1999).

32. Clausen, B. E., Burkhardt, C., Reith, W., Renkawitz, R. \& Förster, I. Conditional gene targeting in macrophages and granulocytes using LysMcre mice. Transgenic Res. 8, 265-277 (1999).

33. Faust, N., Varas, F., Kelly, L. M., Heck, S. \& Graf, T. Insertion of enhanced green fluorescent protein into the lysozyme gene creates mice with green fluorescent granulocytes and macrophages. Blood 96, 719-726 (2000).

34. Jakubzick, C. et al. Lymph-migrating, tissue-derived dendritic cells are minor constituents within steady-state lymph nodes. J. Exp. Med. 205, 2839-2850 (2008).

35. Wantha, S. et al. Neutrophil-derived cathelicidin promotes adhesion of classical monocytes. Circ. Res. 112, 792-801 (2013).

36. Jakubzick, C. et al. Minimal differentiation of classical monocytes as they survey steady-state tissues and transport antigen to lymph nodes. Immunity 39, 599-610 (2013).

37. Limbourg, A. et al. Notch ligand Delta-like 1 is essential for postnatal arteriogenesis. Circ. Res. 100, 363-371 (2007).

38. Kusumbe, A. P., Ramasamy, S. K. \& Adams, R. H. Coupling of angiogenesis and osteogenesis by a specific vessel subtype in bone. Nature 507, 323-328 (2014).

39. Ehling, M., Adams, S., Benedito, R. \& Adams, R. H. Notch controls retinal blood vessel maturation and quiescence. Development 140, 3051-3061 (2013).

40. Benedito, R. et al. Notch-dependent VEGFR3 upregulation allows angiogenesis without VEGF-VEGFR2 signalling. Nature 484, 110-114 (2012).

41. Thomas, G., Tacke, R., Hedrick, C. C. \& Hanna, R. N. Nonclassical patrolling monocyte function in the vasculature. Arterioscler. Thromb. Vasc. Biol. 35, 1306-1316 (2015).

42. Hrabe de Angelis, M., McIntyre, 2nd J. \& Gossler, A. Maintenance of somite borders in mice requires the Delta homologue DII1. Nature 386, 717-721 (1997).

43. Kusumbe, A. P., Ramasamy, S. K. \& Adams, R. H. Coupling of angiogenesis and osteogenesis by a specific vessel subtype in bone. Nature 507, 323-328 (2015)
44. Ehling, M., Adams, S., Benedito, R. \& Adams, R. H. Notch controls retinal blood vessel maturation and quiescence. Development 140, 3051-3061 (2015)

45. Koch, U. et al. Delta-like 4 is the essential, nonredundant ligand for Notch1 during thymic T cell lineage commitment. J. Exp. Med. 205, 2515-2523 (2008).

46. Satpathy, A. T. et al. Notch2-dependent classical dendritic cells orchestrate intestinal immunity to attaching-and-effacing bacterial pathogens. Nat. Immunol. 14, 937-948 (2013).

47. Ishifune, C. et al. Differentiation of CD11c + CX3CR1 + cells in the small intestine requires Notch signaling. Proc. Natl Acad. Sci. USA 111, 5986-5991 (2014).

48. Ye, M. et al. Hematopoietic stem cells expressing the myeloid lysozyme gene retain long-term, multilineage repopulation potential. Immunity 19, 689-699 (2003).

49. Limbourg, A. et al. Evaluation of postnatal arteriogenesis and angiogenesis in a mouse model of hind-limb ischemia. Nat. Protoc. 4, 1737-1746 (2009).

50. Schmittgen, T. D. \& Livak, K. J. Analyzing real-time PCR data by the comparative C(T) method. Nat. Protoc. 3, 1101-1108 (2008).

\section{Acknowledgements}

We thank Sylvana Henschen and Stefan Sablotny for excellent technical assistance; Achim Gossler for Dll1 $1^{+/ l a c Z}$ mice; the Central Animal Facility, Research Core Facility Cell Sorting, Research Core unit Laser Microscopy of Hannover Medical School for excellent support. This research has been funded by grants from DFG (Li948-5/1), BMBF (01GU1105B), IFB-Tx (01EO1302) and GIF (1089) to F.P.L. and SFB1054-B04 to C.W

\section{Author contributions}

J.G., R.G., J.J., O.S., J.D., C.G.B., K.K., A.L., C.H., T.K., S.K.R., C.I., R.H., L.C.N. did the experiments; J.G., R.G., J.J., O.S., C.H., J.D., C.G.B., C.I., K.Y., F.P.L. designed and analysed experiments; J.G., F.R., L.J.S., U.Z.S., J.B., H.H., R.H.A., C.W., K.M.M., K.Y., F.P.L. wrote and edited the manuscript; F.P.L. conceived the research and directed the study.

\section{Additional information}

Supplementary Information accompanies this paper at http://www.nature.com/ naturecommunications

Competing financial interests: The authors declare no competing financial interests.

Reprints and permission information is available online at http://npg.nature.com/ reprintsandpermissions/

How to cite this article: Gamrekelashvili, J. et al. Regulation of monocyte cell fate by blood vessels mediated by Notch signalling. Nat. Commun. 7:12597 doi: 10.1038/ncomms12597 (2016).

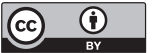

This work is licensed under a Creative Commons Attribution 4.0 International License. The images or other third party material in this article are included in the article's Creative Commons license, unless indicated otherwise in the credit line; if the material is not included under the Creative Commons license, users will need to obtain permission from the license holder to reproduce the material. To view a copy of this license, visit http://creativecommons.org/licenses/by/4.0/

C) The Author(s) 2016 


\section{Corrigendum: Regulation of monocyte cell fate by blood vessels mediated by Notch signalling}

Jaba Gamrekelashvili, Roberto Giagnorio, Jasmin Jussofie, Oliver Soehnlein, Johan Duchene, Carlos G. Briseño, Saravana K. Ramasamy, Kashyap Krishnasamy, Anne Limbourg, Christine Häger, Tamar Kapanadze, Chieko Ishifune, Rabea Hinkel, Freddy Radtke, Lothar J. Strobl, Ursula Zimber-Strobl, L. Christian Napp, Johann Bauersachs, Hermann Haller, Koji Yasutomo, Christian Kupatt, Kenneth M. Murphy, Ralf H. Adams, Christian Weber \& Florian P. Limbourg

Nature Communications 7:12597 doi: 10.1038/ncomms12597 (2016); Published 31 Aug 2016; Updated 3 May 2017

The authors inadvertently omitted Christine Häger, who was involved in the initial characterization of Notch mutant mice presented in this Article, from the author list and Author contributions statement. These errors have now been corrected in both the PDF and HTML versions of the Article.

(c) (i) This work is licensed under a Creative Commons Attribution 4.0 International License. The images or other third party material in this article are included in the article's Creative Commons license, unless indicated otherwise in the credit line; if the material is not included under the Creative Commons license, users will need to obtain permission from the license holder to reproduce the material. To view a copy of this license, visit http://creativecommons.org/licenses/by/4.0/

(C) The Author(s) 2017 